\title{
Cortical dynamics during naturalistic sensory stimulations: Experiments and models
}

\author{
Alberto Mazzoni ${ }^{\text {a }}$, Nicolas Brunel ${ }^{\text {b,c,d }}$, Stefano Cavallari ${ }^{\text {a }}$, Nikos K. Logothetis ${ }^{\text {d,e }}$, Stefano Panzeri ${ }^{\text {a,* }}$ \\ ${ }^{a}$ Robotics, Brain and Cognitive Sciences Department, Italian Institute of Technology, Via Morego 30, 16163 Genova, Italy

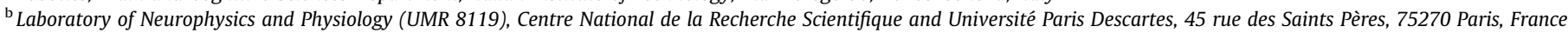 \\ ${ }^{\mathrm{C}}$ Institute for Scientific Interchange, viale Settimio Severo 65, 10133 Turin, Italy \\ ${ }^{\mathrm{d}}$ Max Planck Institute for Biological Cybernetics, Spemannstrasse 38, 72076 Tübingen, Germany \\ ${ }^{\mathrm{e}}$ Division of Imaging Science and Biomedical Engineering, University of Manchester, Manchester M13 9PT, United Kingdom
}

\section{A R T I C L E I N F O}

\section{Keywords:}

Vision

Recurrent networks

Integrate and fire

LFP modeling

Neural code

Spike timing

Natural movies

Mutual information

Oscillations

Gamma band

\begin{abstract}
A B S T R A C T
We report the results of our experimental and theoretical investigations of the neural response dynamics in primary visual cortex (V1) during naturalistic visual stimulation. We recorded Local Field Potentials (LFPs) and spiking activity from V1 of anaesthetized macaques during binocular presentation of Hollywood color movies. We analyzed these recordings with information theoretic methods, and found that visual information was encoded mainly by two bands of LFP responses: the network fluctuations measured by the phase and power of low-frequency (less than $12 \mathrm{~Hz}$ ) LFPs; and fast gamma-range (50$100 \mathrm{~Hz}$ ) oscillations. Both the power and phase of low frequency LFPs carried information largely complementary to that carried by spikes, whereas gamma range oscillations carried information largely redundant to that of spikes. To interpret these results within a quantitative theoretical framework, we then simulated a sparsely connected recurrent network of excitatory and inhibitory neurons receiving slowly varying naturalistic inputs, and we determined how the LFPs generated by the network encoded information about the inputs. We found that this simulated recurrent network reproduced well the experimentally observed dependency of LFP information upon frequency. This network encoded the overall strength of the input into the power of gamma-range oscillations generated by inhibitory-excitatory neural interactions, and encoded slow variations in the input by entraining the network LFP at the corresponding frequency. This dynamical behavior accounted quantitatively for the independent information carried by high and low frequency LFPs, and for the experimentally observed cross-frequency coupling between phase of slow LFPs and the power of gamma LFPs. We also present new results showing that the model's dynamics also accounted for the extra visual information that the low-frequency LFP phase of spike firing carries beyond that carried by spike rates. Overall, our results suggest biological mechanisms by which cortex can multiplex information about naturalistic sensory environments.
\end{abstract}

(c) 2011 Elsevier Ltd. All rights reserved.

\section{Introduction}

The natural visual environment is complex and contains many features (such as the color, shape or orientation of images and objects) which continuously change over a wide range of spatial and temporal scales (Simoncelli, 2003). To understand how sensory areas of cortex cope with the demands of representing such a complex environment, it is important to address two questions. The first question regards what type of neural response dynamics is best suited to represent the complexity of the visual stimuli and their evolution over time. The second question regards the mechanisms underlying the generation of such dynamics, i.e. what are

\footnotetext{
* Corresponding author. Tel.: +39010 71781437.

E-mail address: stefano.panzeri@iit.it (S. Panzeri).
}

the biological processes translating the visual stimuli into the neural responses.

In order to answer the first question, a key step is to record neural responses of cortical visual areas to naturalistic visual stimuli, which replicate at least part of the complexity of the natural environment. Using naturalistic stimuli has also limitations (which mostly reflect the difficulty of the interpretation of their results (Rust and Movshon, 2005)) and cannot replace the knowledge gained by decomposing neural dynamics in terms of the effect of simple carefully controlled stimulus parameters (Benucci et al., 2009). However, analyzing neural responses to stimuli such as color movies which have a complex spatio-temporal structure similar of the natural world (Geisler, 2008) is important for several reasons. First, naturalistic stimuli are likely to engage some aspects of neural response which may not be elicited in the presence of simpler stimuli (Felsen and Dan, 2005; Reinagel, 2001). For exam- 
ple, any neural representation of stimulus time is unlikely to be engaged by stimuli with quasi stationary or impoverished dynamics. Moreover, responses to naturalistic visual stimuli have been found to be more reliable (Hasson et al., 2009), suggesting that coding in the visual system may be optimized for processing naturalistic stimuli (Dan et al., 1996).

While earlier neurophysiological work has concentrated in characterizing spiking responses to naturalistic stimuli (Baddeley et al., 1997; Desbordes et al., 2008; Smyth et al., 2003; Treves et al., 1999), more recent work is beginning to complement the analysis of spiking responses with that of Local Field Potentials (LFPs). LFPs are highly localized signals (Berens et al., 2008; Katzner et al., 2009), which relate well to subthreshold integrative processes in areas such as the dendrite which are otherwise inaccessible by recording only spiking activity of a few neurons (Gustafsonn, 1984; Harada and Takahashi, 1983; Kamondi et al., 1998; Logothetis, 2003), and present a rich and easily measurable spectrum of response frequencies (Buszaki, 2006). For this reason, inclusion of LFPs into the analysis of neural responses can offer additional insights into the origin of sensory representations beyond those offered by only measuring neuronal spike trains, because the latter can only give information about the output of the local neural computation implementing the representation of sensory events (Belitski et al., 2010; Nicolelis and Lebedev, 2009; Panzeri et al., 2010).

In order to shed light on the candidate mechanisms underlying information processing, it is useful to complete these studies with models and simulations which can be used to test quantitatively specific hypotheses against the empirical observations. In the last two decades simulations and experiments have built on each other to provide descriptions of a variety of cortical phenomena (Amit et al., 1994; Breakspear et al., 2010; David et al., 2009; Fix et al., 2007).

Here we review a series of neurophysiological (Belitski et al., 2008; Montemurro et al., 2008) and modeling (Mazzoni et al., $2008,2010)$ studies from our group which investigated, respectively through extracellular recordings from the primary visual cortex of anaesthetized macaques and through simulations of recurrent networks, how naturalistic movie stimuli are encoded in different frequency bands of primary visual cortical LFPs. We then extend the previous modeling results to demonstrate that our recurrent network model can account for the additional information about visual stimuli carried by the phase of low frequency LFPs at which spikes are fired, which was described in previous experimental reports (Montemurro et al., 2008). We finally discuss how models and experiments contributed to give a coherent understanding of all these observed phenomena.

\section{Material and methods}

\subsection{Neurophysiological procedures}

We briefly summarize the experimental procedures used to record neural responses to naturalistic color movies in primary visual cortex (V1). We refer to previous studies (Belitski et al., 2008, 2010; Montemurro et al., 2008) for full details.

Four adult rhesus monkeys (Macaca mulatta) participated in the experiments. All procedures were approved by the local authorities (Regierungsprsidium) and were in full compliance with the guidelines of the European Community (EUVD 86/609/EEC) for the care and use of laboratory animals. Prior to the experiments, form-fitted head posts and recording chambers were implanted during an aseptic and sterile surgical procedure (Logothetis et al., 2002). Recordings were obtained while the animals were anaesthetized. We maintained anesthesia with remifentanil $(0.5-2 \mu \mathrm{g} / \mathrm{kg} / \mathrm{min})$ in combination with a fast acting paralytic, mivacurium chloride (5-7 $\mathrm{mg} / \mathrm{kg} / \mathrm{h})$. Body temperature was strictly maintained at 38$39{ }^{\circ} \mathrm{C}$, and end-tidal $\mathrm{CO} 2$ and oxygen saturation were kept constant at $33 \mathrm{~mm} \mathrm{Hg}$ and over 95\%, respectively. Acidosis was prevented by the administration of lactated Ringer's solution with $2.5 \%$ glucose, infused at $10 \mathrm{ml} / \mathrm{kg} / \mathrm{h}$, and intravascular volume was maintained by the additional administration of colloids (hydroxyethyl starch, 20-30 ml over $1-2 \mathrm{~min}$ or $20 \mathrm{ml} / \mathrm{kg} / \mathrm{h}$ ). To ensure that no stress was caused to the animal, we measured catecholamines and optimized dosages to ensure unaffected physiological responses at normal catecholamine concentrations (Logothetis et al., 1999).

The main reason for collecting neural responses during anesthesia is that this protocol offers several advantages for the investigation of primary cortical dynamics. In particular: microcircuits can be studied in a "idle-state" without the strong effects of animal state, including effects of attention and arousal that introduce additional complication in the interpretation of signals; some practical issues, such as those arising from trial to trial variations of eye movements, are simply eliminated; the initial correct interpretation of the basic functional profile of neural circuits requires excellent signal-to-noise ratio, which can be more easily obtained in anesthetized animals due to long acquisition times. The main reason for using opiates was the fact that they selectively act on the pain matrix while largely sparing sensory areas. The concentration of opiate receptors, i.e. $\mu, \kappa, \sigma$ and $\delta$ (Pfeiffer et al., 1982), varies over different brain regions (Leppa et al., 2006), with high concentrations mainly found in the so-called pain matrix (Talbot et al., 1991). The latter refers to the regions commonly activated in response to noxious stimulation, namely thalamus, somatosensory/ motor cortices, insula, cingulate cortex, basal ganglia, as well as orbitofrontal, frontal and parietal cortices. Remifentanil, an ultrafast-acting $\mu$-opioid receptor agonist, has no significant effect on the neurovascular and neural activity of brain areas that do not belong to the pain matrix (Goense et al., 2008; Goense and Logothetis, 2008; Logothetis et al., 1999, 2009; Lund et al., 1994; Sereno et al., 2002; Zappe et al., 2008a,b). The visual cortex in particular does not even bind remifentanil (Jones et al., 1991), and early visual information processing is largely preserved during remifentanil anesthesia (Lund et al., 1994).

Neuronal activity was recorded from opercular V1 (foval and para-foveal representations) using microelectrodes (FHC Inc., Bowdoinham, Maine, $300-800 \mathrm{k} \Omega$ ) which were arranged in a $4 \times 4$ square matrix (interelectrode spacing varied from $1 \mathrm{~mm}$ to $2.5 \mathrm{~mm}$ ) and introduced in each experimental session into the cortex through the overlying dura mater by a microdrive array system (Thomas Recording, Giessen, Germany). Electrode tips were typically positioned in the upper or middle cortical layers. In total we recorded from 55 sites in V1 with a well-defined receptive field within the field of movie projection.

Visual stimuli were presented binocularly at a resolution of $640 \times 480$ pixels (field of view: $30 \times 23^{\circ}, 24$ bit true color, $60 \mathrm{~Hz}$ refresh) using a fiberoptic system (Avotec, Silent Vision, Florida). Stimuli consisted of 'naturalistic' complex and commercially available Hollywood movies ( $30 \mathrm{~Hz}$ frame rate), from which 3.5-6 min long sequences were presented and repeated 30-40 times. The receptive fields of all recording sites analyzed were within the area of visual stimulation (Rasch et al., 2008).

\subsubsection{Spectral separation and spectral analysis of extracellular signals}

Extracellularly recorded neural signals were amplified using an Alpha Omega amplifier system (Alpha Omega GmbH, Ubstadt-Weiher, Germany) and recorded at $20.83 \mathrm{kHz}$. The amplifying system filtered out the frequencies below $1 \mathrm{~Hz}$. The LFPs were extracted from these raw recordings by low-pass-filtering the neural signal in the frequency range up to $250 \mathrm{~Hz}$. The LFP extraction procedure lowpass filtered and downsampled the neural signal to a rate of 
$500 \mathrm{~Hz}$ using a Kaiser filter with bandwidth 1-250 Hz, sharp transition bandwidth $(1 \mathrm{~Hz})$, very small passband ripple $(0.01 \mathrm{~dB})$, and high stopband attenuation $(60 \mathrm{~dB})$. Forwards and backwards filtering was used to eliminate phase shifts introduced by the filters. In order to extract multiunit spike times, the $20.83 \mathrm{kHz}$ neural signal was filtered in the high-frequency range of $500-3500 \mathrm{~Hz}$. The threshold for spike detection was set at 3.5 standard deviations. A spike was recognized as such only if the last spike occurred more than $1 \mathrm{~ms}$ earlier. For the present analysis we did not separate single and multi-units.

The band-limited LFP signals were generated using the same Kaiser filters described above with the appropriate bandwidth settings. From these band-passed signals, we computed the instantaneous phase as the argument of the Hilbert transform.

To assess how the power of LFP and spike rate oscillations changed over different segments of the movies, we divided each movie into non-overlapping time windows of length $T$ (a parameter that was varied in a wide range (Belitski et al., 2008)). The resulting structure of the neural activity in each stimulus time window $s$ was quantified by computing the power spectrum $r_{f}$ at each frequency $f$, independently for each trial using the multitaper technique (Percival and Walden, 1993) which provides an efficient way to simultaneously control the bias and variance of spectral estimation.

\subsection{Model}

Here we briefly describe the methods used to simulate spiking and LFP responses of recurrent networks of excitatory and inhibitory neurons. The model was first introduced by Brunel (2000) and Brunel and Wang (2003)). It was later extended (Mazzoni et al., 2008, 2010) to include time-varying inputs. We summarize here the structure of the network and we refer to the original reports (Mazzoni et al., 2008, 2010) for full details.

The simulated network was composed of 4000 pyramidal neurons with AMPA-like synapses, and 1000 interneurons with GABA-like synapses. The network connectivity was random and sparse, with a connection probability of 0.2 between any directed pair of cells. Both pyramidal neurons and interneurons were described by voltage-based leaky integrate and fire (LIF) dynamics with fixed threshold, fixed refractory time (Tuckwell, 1988). The model included conduction delays and a post-spike refractory period. The AMPA and GABA postsynaptic currents were determined by the spikes emitted by the pre-synaptic neurons of the network and by the external inputs. Compound synaptic currents were the linear sum of contributions induced by single pre-synaptic spikes. Each contribution was described by a difference of exponentials, with rise time, decay time, and amplitude depending on the kind of synapse and the category of the post-synaptic neuron. All values were of the order of magnitude of the values reported in the literature (Gupta et al., 2000; Markram et al., 1997). We verified that modifying these values did not affect qualitatively the results as long as the network was in the weakly synchronized regime. Each neuron received inhibitory and excitatory inputs from the neurons of the network, and also received two types of distinct external excitatory drives (see Fig. 1A). A "thalamic" input carried the simulated sensory information, and was injected through synapses with timescales and strength resembling those of thalamocortical synapses (Gil and Amitai, 1996). An "unspecific" input represented instead stimulus unrelated changes of ongoing activity and nonspecific contributions from other areas of cortex and was injected through AMPA synapses identical to those of the network. Since cortical ongoing activity has the most power in the slow frequency range, these non-specific contributions were generated according to an Orstein-Uhlenbeck process with a low pass cut-off frequency of $10 \mathrm{~Hz}$. Synapses carrying both types of external inputs were acti- vated by random Poisson spike trains, with time-varying rates which were identical for all neurons.

Three types of inputs signal were injected, in different simulation sessions, from the model "thalamic" region: (i) time-invariant ("constant") inputs, (ii) perfectly periodic inputs which varied sinusoidally in time, (iii) "naturalistic" input spike trains which reproduced the firing activity recorded in the LGN of an anaesthetized monkey during one of the binocular naturalistic visual stimulation sessions described in Section 2.1 (Rasch et al., 2008).

Since a prominent contribution to real cortical LFPs arises from current flows due to synaptic activity (Logothetis, 2003; Mitzdorf, 1985), we computed the simulated LFP signal generated by the network as the sum of the absolute values of AMPA and GABA currents (Buehlmann and Deco, 2008; Mazzoni et al., 2008, 2010). We decided to sum the absolute values of currents because AMPA synapses are usually apical and GABA synapses are usually peri-somatic and thus their dipoles sum with the same sign along the dendrite (Fig. 1B). In computing the simulated LFPs, we summed only currents from synapses of pyramidal neurons (Fig. 1B), under the assumption that pyramidal neurons contribute more due to their approximate open field arrangement (Murakami and Okada, 2006). In Mazzoni et al. (2010) the LFP signal was taken with a negative sign to be directly comparable with experimental recordings. Although more detailed quantifications of signals such as LFPs from simulated networks are in principle possible (see e.g. Pettersen et al., 2008), we found that the simple quantification we chose was enough to reproduce all the features of interest of experimentally recorded LFPs (see Sections 3.2 and following), thereby suggesting that our simple description of LFPs was sufficient for the present purpose.

\subsection{Information analysis}

To quantify the stimulus selectivity of neural responses, we measured the mutual information between a given set of stimuli $S$ and a set of neural responses $R$. Mutual information (abbreviated as "information" in the following), is a popular measure of the goodness of stimulus encoding in neuroscience (de Ruyter van Steveninck et al., 1997; Fairhall et al., 2001; Panzeri et al., 2003). It quantifies the reduction of the uncertainty about the stimulus that can be gained from observing a single-trial neural response, and we measured it in units of bits ( 1 bit means a reduction of uncertainty by a factor of two).

Information depends on both the choice of the stimulus set and of the quantification of the neural response. To create the stimulus set, we divided the presentation time of the dynamic stimulus (a movie in the case of recordings from visual cortex, and a time dependent input spike train in the case of neural network simulations) into different segments of length $T$ (a parameter that was varied in the range from few ms to several seconds) and each segment was considered as a different stimulus $s$ (see schematic in Fig. 2). We then computed the information about which dynamic stimulus time segment elicited the considered response. This procedure has several advantages. The first is that it is simple to apply and lends itself to comparisons between experimental and theoretical data. The second is that it does not make any assumption as to which specific features of the dynamic stimulus triggered the neural response and so can potentially capture the information about all possible dynamical stimulus features presented experimentally (de Ruyter van Steveninck et al., 1997). Regarding the choice of neural response, we considered several different possibilities, as detailed next.

We first computed the information between the stimulus and the power of the LFP at a given frequency $f$, as follows.

$$
I\left(S ; R_{f}\right)=\sum_{s} P(s) \sum_{r_{f}} P\left(r_{f} \mid s\right) \log _{2} \frac{P\left(r_{f} \mid s\right)}{P\left(r_{f}\right)}
$$



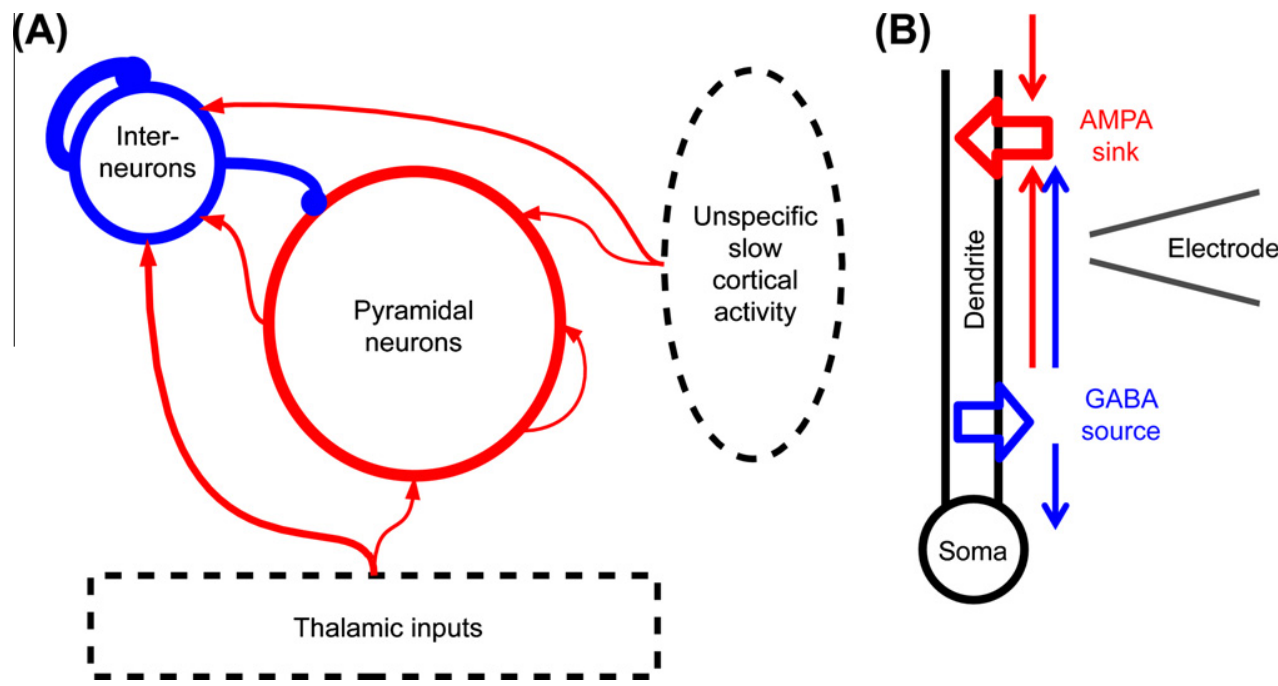

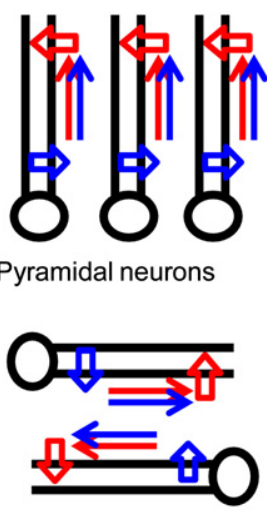

Interneurons

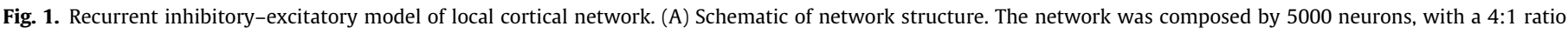

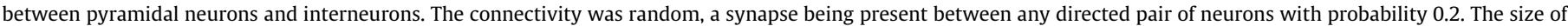

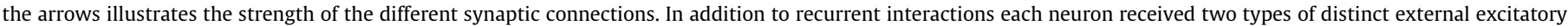

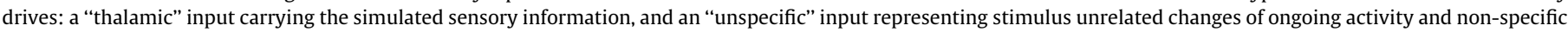

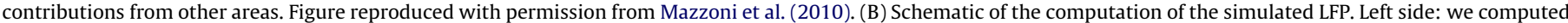

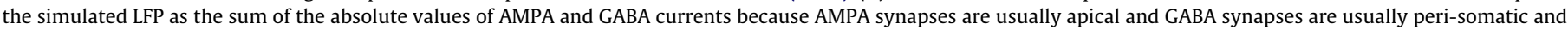

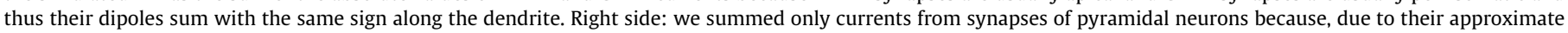
open field arrangement, they contribute to LFP more than interneurons, which have a much less regular dendritic spatial organization.

where $P(s)$ is the probability of presentation of the stimulus window $s$ (here equal to the inverse of the total number of stimulus windows (Fig. 2A)), $P\left(r_{f} \mid s\right)$ is the probability of observing a power $r_{f}$ at frequency $f$ in response to a single trial to stimulus $s$ (Fig. 2D and $\mathrm{E}$ ), and $P\left(r_{f}\right)$ is the probability of observing power $r_{f}$ across all trials in response to any stimulus (Fig. 2C). To facilitate the sampling of response probabilities, the space of power values at each frequency was binned into six equipopulated bins (Belitski et al., 2008). For all measures of information about power presented here, we used a stimulus window of length $T=2.048 \mathrm{~s}$.

The above single-frequency information analysis was extended to compute how much information about the stimuli we can obtain when combining together the power $r_{f 1}$ and $r_{f 2}$ at two different frequencies. The mutual information that the joint knowledge of the powers $r_{f 1}$ and $r_{f 2}$ conveys about the stimulus is defined as:

$$
I\left(S ; R_{f 1} R_{f 2}\right)=\sum_{s} P(s) \sum_{r_{f 1} r_{f 2}} P\left(r_{f 1} r_{f 2} \mid s\right) \log _{2} \frac{P\left(r_{f 1} r_{f 2} \mid s\right)}{P\left(r_{f 1} r_{f 2}\right)}
$$

where the stimulus-response probabilities defined in Eq. (1.2) are analogous to the ones in Eq. (1.1) - see Belitski et al. (2008) for full details. The redundancy between the information carried by the powers at the two considered frequencies is defined as the difference between the sum of the information carried by the power of each frequency individually and their joint information:

$\operatorname{Red}\left(S ; R_{f 1} R_{f 2}\right)=I\left(S ; R_{f 1}\right)+I\left(S ; R_{f 2}\right)-I\left(S ; R_{f 1} R_{f 2}\right)$

We finally considered the information carried by phase of firing code, which is the neural response defined when the timing of spikes emitted in response to the stimulus are measured with respect to the phase of a concurrent LFP wave bandpassed around a considered frequency $f$. This can be done by dividing the phase range into quarters, and then by tagging the spikes with a label indicating the phase quadrant at which they were emitted. Then, the phase of firing information can be defined as follows:

$I\left(S ; R_{f}^{\Phi}\right)=\sum_{s} P(s) \sum_{\varphi_{f}=0}^{4} P\left(\varphi_{f} \mid s\right) \log _{2} \frac{P\left(\varphi_{f} \mid s\right)}{P\left(\varphi_{f}\right)}$ where in the above equation the response set is composed by five different symbols: $\varphi_{f}=0$ denotes the absence of spikes in response to the stimulus in the considered trial, and $\varphi_{f}=1,2,3,4$ denote that a spike was emitted when the LFP phase was in a given quadrant. To evaluate if the phase of firing carries information above and beyond that carried by spike rates, we compared $I\left(S ; R_{f}^{\Phi}\right)$ to the spike rate information, which was evaluated from Eq. (1.4) but after randomly shuffling the responses $\varphi_{f}>0$ independently for each trial. The shuffling operation preserves all the information carried by the spike rate while at the same time completely destroying any additional information that may be carried by the knowledge of phase. For all phase of firing information measures reported here, we used a stimulus window $T$ of $4 \mathrm{~ms}$ (Montemurro et al., 2008).

In computing all the above information quantities, all the stimulus-response probabilities were evaluated empirically from all available stimulus repetitions ("trials"). Since the number of available trials may be limited (and especially so when considering real rather than simulated data), estimation of probabilities suffers from statistical errors which lead to a systematic error in information estimates (called limited sampling bias). We corrected for this problem using the procedures reported in Refs. Montemurro et al. (2007a,b) and Panzeri et al. (2007) and implemented in the Information Breakdown Toolbox (www.ibtb.org, Magri et al., 2009) which we used for the analysis.

\subsection{Circular variance analysis of entrainment}

The strength by which a given frequency component of the LFP of the simulated network entrained to a given frequency component of the time course of the network spike rate input was quantified by the circular variance over time of the phase difference between the considered frequency component of the LFP and the considered frequency component of the input (each bandpassed in $2 \mathrm{~Hz}$ wide bands with the Kaiser filter detailed above). Circular variance, defined e.g. in Fisher (1993) is a measure of the dispersion of a distribution of angles and in our case ranges from 0 (perfect phase locking and entrainment) to 1 (no entrainment). 

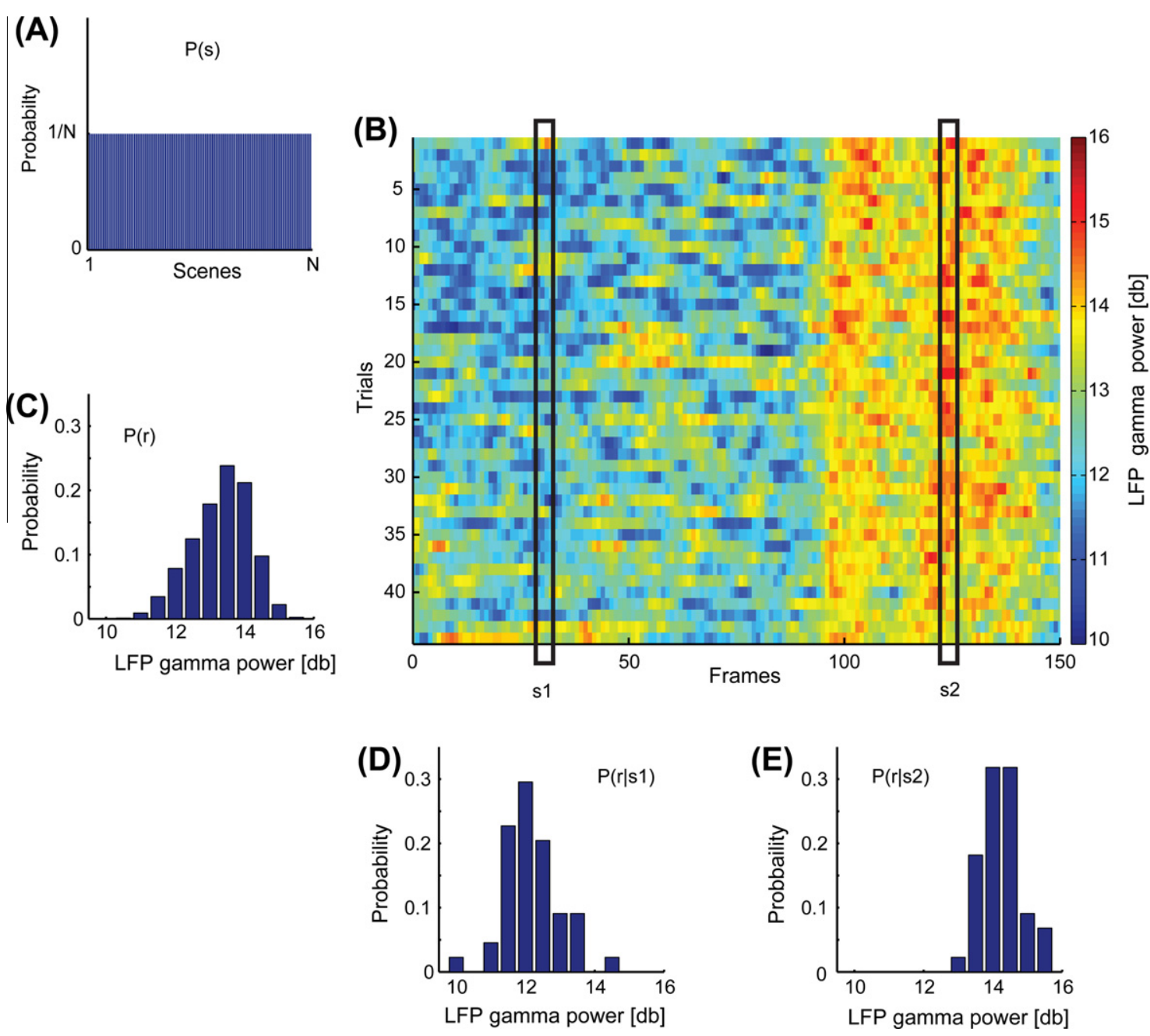

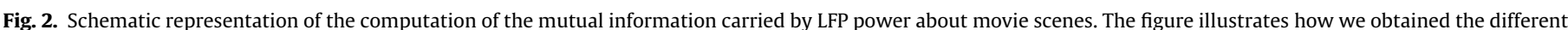

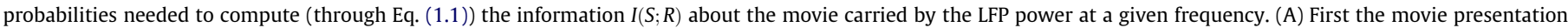

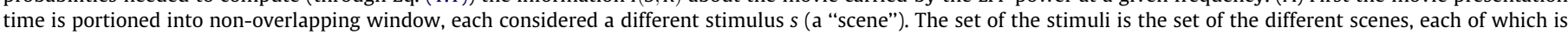

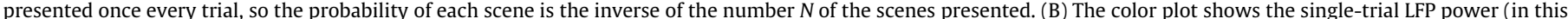

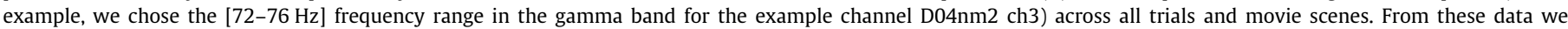

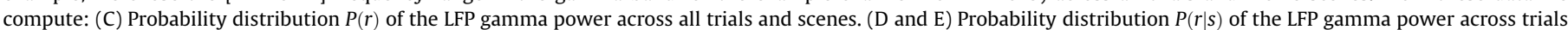

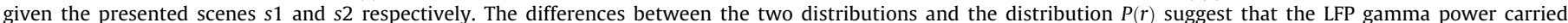
information about which scene is presented. Computing $P(r \mid s)$ for all scenes and inserting it in Eq. (1.1) gave the actual value of the mutual information.

\section{Information content of Local Field Potential during naturalistic stimulations}

\subsection{LFP recordings in response to natural stimuli suggest that cortex multiplexes information over a small number of different frequency bands}

Recordings of LFPS from cortical areas indicate that sensory cortex responds to sensory stimuli with a very broad spectrum of fluctuations, which spans a wide range of frequencies ranging from less than one $\mathrm{Hz}$ to hundred $\mathrm{Hz}$ or more (Buszaki, 2006; Kayser and Konig, 2004; Lakatos et al., 2005; Senkowski et al., 2007). However, the functional consequences and the implications for sensory coding of this wide range of frequencies remain largely unknown. On the one hand, the presence of a wide spectrum of activity could imply that there is no privileged scale for information representation, because information is evenly spread over all scales. This view is consistent with the proposal that neural activity is largely scale free (Bedard et al., 2006; He et al., 2010; Mazzoni et al., 2007). On the other hand, it is possible that information is represented by only a small number of specific frequency ranges, each carrying a separate contribution to the information representation.

To shed light on this issue, it is important to quantify the information content of each frequency range of neural activity, and understand which ranges carry complementary or similar information. To investigate this question, we used spectral analysis and information theory to analyze LFPs and spiking responses recorded from the primary visual cortex of anaesthetized macaques in response to binocularly-presented naturalistic color movies (Belitski et al., 2008).

To quantify the characteristics of LFP fluctuations at different frequencies, we first computed the LFP spectrum during the entire period of movie presentation (reported for an example channel in Fig. 3A). The LFP spectrum had a very wide band with fluctuations ranging over the entire frequency range analyzed. The highest LFP power was at low frequencies $(<12 \mathrm{~Hz})$, and the power decreased steeply at increasing frequencies. We compared the averaged LFP spectrum evoked during the movie to the LFP spectrum of the same electrode during spontaneous activity (measured in the absence of visual stimulation). There was an increase of power during movie stimulation at frequencies below $12 \mathrm{~Hz}$ (Fig. 3A). The evoked and 

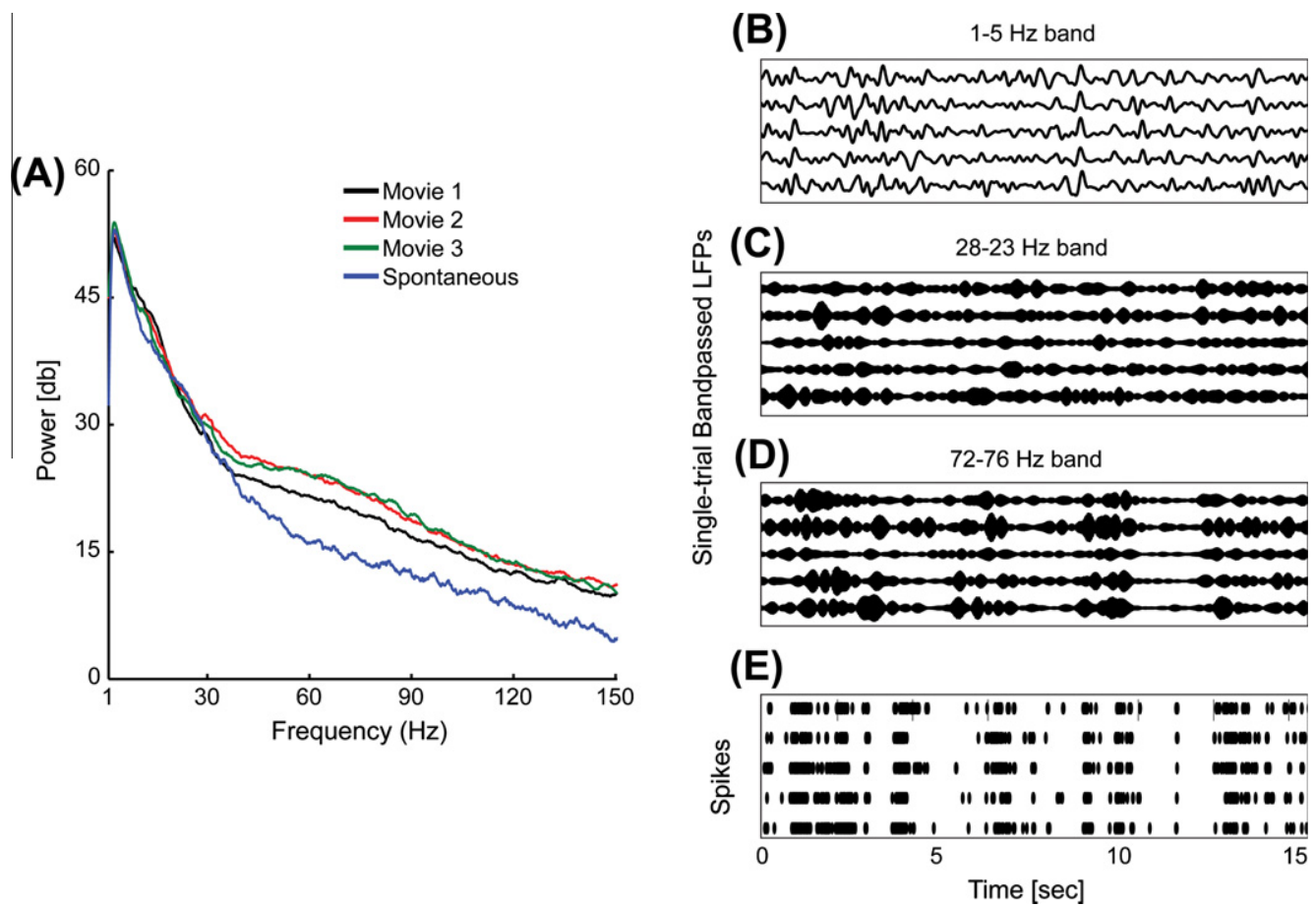

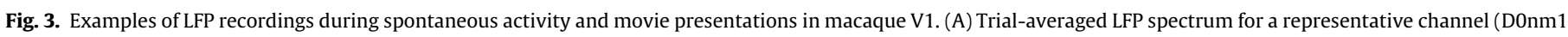

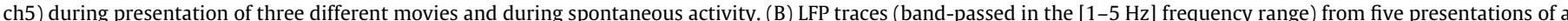

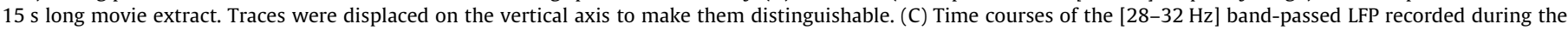

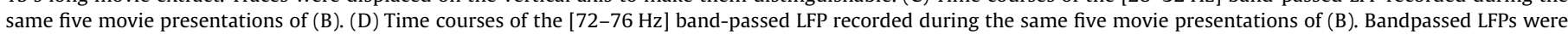

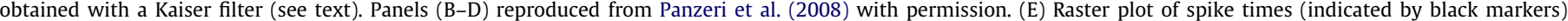
recorded from the same electrode of LFP displayed in (B-D) during the same five presentations of (B).

spontaneous LFP spectrograms were similar at frequencies between 12 and $24 \mathrm{~Hz}$, while the power associated to frequencies $>24 \mathrm{~Hz}$ was higher during movie presentation. Consistent with previous studies (Henrie and Shapley, 2005), we found the most substantial power increase over spontaneous activity of the movieevoked LFP in the gamma frequency region [30-100 Hz].

We next analyzed how the neural signals responded to the movie. Fig. 3B-D shows the bandpassed LFP responses (in the [1$5 \mathrm{~Hz}],[28-32 \mathrm{~Hz}]$ and [72-76 Hz] frequency range respectively) for several trials, from a representative example recording site during the presentation of a 15 -s long movie sequence. The presentation of the movie elicited LFP patterns that, both in the low frequency $(1-5 \mathrm{~Hz})$ range (Fig. 3B) and in the [72-76 Hz] range within the high gamma region (Fig. 3D), were clearly modulated by the movie and repeatable across trials: episodes of high instantaneous power were elicited reliably in correspondence of certain scenes in the movie. In contrast, LFP waveforms in the intermediate frequency range [28-32 Hz] (Fig. 3C) could not be reliably associated to the movie time course.

We also investigated the $\mathrm{V} 1$ spiking responses to the movie. Fig. 3E shows that the spike rates clearly encoded the movie. The high spike rate episodes were associated more closely with episodes of high LFP power in the high-gamma LFP frequency range than at lower LFP frequencies, suggesting that gamma LFPs may be more closely related to the stimulus-modulated spiking activity than low LFP frequencies.

We then investigated more quantitatively how the power of different LFP frequency bands encoded the stimuli computing the information that the LFP power carried about which scene was being presented (see Eq. (1.1) and Section 2.3). The information of the LFP power, averaged over all channels recorded in session A98 (the one selected for comparison with model results, see Sections 2.2 and 3.2), is reported in Fig. 4A. We found two informative bands in the LFP spectrum: a low frequency range below $10 \mathrm{~Hz}$ (corresponding to the delta and theta bands) and a high frequency range $[50-100 \mathrm{~Hz}]$ in the gamma band.

Having established that both high-gamma and low frequencies of LFPs convey information, the next important question is to establish whether the different informative frequencies ranges are redundant or not, i.e. whether they carry the same or different stimulus information. Thus, we computed both the joint information carried by the power of pairs of LFP frequencies (Eq. (1.2)) and their redundancy (Eq. (1.3)). The information obtained by the combined knowledge of the power at low frequencies and the power at gamma frequencies was nearly the sum of the information carried by the two frequencies separately (Fig. 4B). This means that the redundancy between the information carried by the power of high and low frequencies is nearly zero. In contrast, frequencies in the gamma band were highly redundant between each other (Fig. 4C), suggesting that all frequencies in the gamma range reflect largely the same network phenomenon. We found also that LFP frequencies below $40 \mathrm{~Hz}$ carried independent information with respect to spike rates, and were indeed totally decoupled from spike rates (both in terms of stimulus selectivity and trial to trial covariations). However, the power of gamma range LFPs was largely (but not completely) redundant to the spike rate, suggesting that spike rates and gamma power are a largely overlapping information channel (results not reported here but fully explained in Belitski et al. (2008)).

In summary, V1 LFP spectral information during naturalistic stimulations is multiplexed in two different and independent streams, one at very low frequencies and one at gamma frequencies. The gamma power carries information partly (but not completely) redundant to that carried by spike rates. Therefore, out of the many time scales of neural activity that have significant power, only a handful of time scales seem to carry different types of information. An advantage of encoding information into a limited number of frequencies is that it gives the benefit of multiplexing without complicating too much the decoding procedure, which 

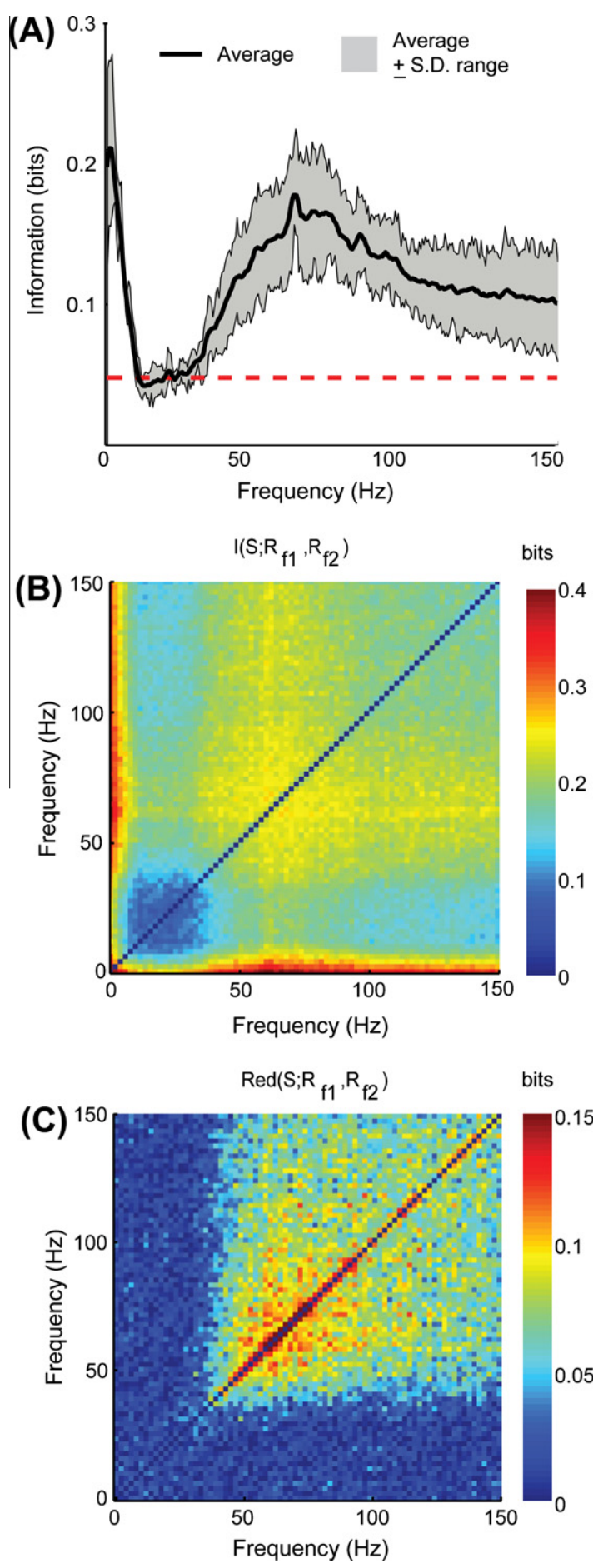

Fig. 4. Information about movie scenes carried by LFP power. (A) Information about the movie scenes carried by the LFP power of experimental session A98. Gray area represents mean \pm STD area across all channels. The red dashed line represents he $p=0.05$ (bootstrap test) significance line of information values. (B) Joint information and $(C)$ redundancy of power for all frequency pairs, averaged over all channels in session A98.

needs to pay attention only to a small number of different frequencies rather than to a continuum of time scales.

\subsection{A recurrent network model reproduces and explains frequency multiplexing of information}

The experimental findings described in the previous section raise two important questions. The first question regards the net- work mechanisms generating these two information channels. The second question regards the nature of the sensory information and of the type of stimulus features encoded in the two bands.

In order to answer these questions, we used a simulated model of a recurrent sparsely connected neural network with excitatory and inhibitory neurons (Mazzoni et al., 2008) and we computed the LFPs generated by these networks under different types of stimulation conditions. These networks are simple, but able to capture the interplay between excitation and inhibition, which is one feature of the organization of cortical microcircuits which is believed to shape the dynamics of local mass activation (Deco et al., 2008; Douglas and Martin, 1991; Logothetis, 2008). The sparse and random network (Fig. 1A) was composed of inhibitory and excitatory neurons and received external excitatory synaptic inputs mimicking the thalamic inputs (conveying the information about sensory stimuli) and the ongoing cortical fluctuations (summarizing the effect of the slow covariations in network state due to ongoing activity). The network LFP was modeled as the sum of the absolute values of AMPA and GABA currents computed on pyramidal neurons (see Section 2.2 and Fig. 1B).

We used different inputs to analyze the response properties of the network. In a first series of simulations in Mazzoni et al. (2008) we injected the network with constant inputs of different intensity. In agreement with previous studies (Brunel and Wang, 2003), we found that the gamma power of the LFP increased monotonically with input strength (Fig. 5A). These results are consistent with neurophysiological findings that grating stimuli of increasing contrast (which is known to modulate the thalamic input to V1 (Derrington and Lennie, 1984; Shapley et al., 1981)) indeed modulate also the power of the LFP gamma band in V1 (Friedman-Hill et al., 2000; Henrie and Shapley, 2005). Similar relationships between stimuli intensity and gamma band power have also been found in non-invasive recordings in humans (Muthukumaraswamy et al., 2010; Swettenham et al., 2009). Furthermore, in the network model (Mazzoni et al., 2008) increases in the inputs intensity were not only associated to a stronger power of the gamma band, but also to an higher peak frequency, consistent with the experimental recordings from visual cortices (Ray and Maunsell, 2010).

In a second set of simulations we studied the response of the network to time-varying inputs. We injected periodic inputs of different frequency and intensity. We found that a sufficiently strong and slow periodic input was able to entrain the network LFP at the corresponding frequency (Fig. 5B and C). This is compatible with the entrainment between low frequency stimuli and neural activity recently observed in auditory cortices (Chandrasekaran et al., 2010; Lakatos et al., 2008; Luo and Poeppel, 2007). We quantified the entrainment measuring the circular variance of the phase difference between the LFP and the inputs for each pair of frequency bands (see Section 2.4). Entrainment was shown to depend not only on the input strength, but also on the input frequency, and was in general stronger at low frequencies of the input (Fig. 5C).

In a third and final set of simulations we injected the network with a naturalistic input based on the firing activity recorded from the LGN of an anaesthetized macaque presented with naturalistic movies (see Section 2.1). The LGN recordings were performed during the same session of the V1 LFP recordings whose spectral information was presented in Fig. 4. The naturalistic LGN spiking response had a spectrum with a strong power associated to a broad range of low frequencies (Fig. 6A). Fig. 6B illustrates that the rules found above for simple inputs held also for the broadband naturalistic input: the low frequency LFP entrained to the large-amplitude slow fluctuations of the input, and the power of gamma oscillations increased in correspondence of high input rates. As a consequence, the information carried by the LFP power of the simulated network during naturalistic stimulation closely matched the experimental 
(A)

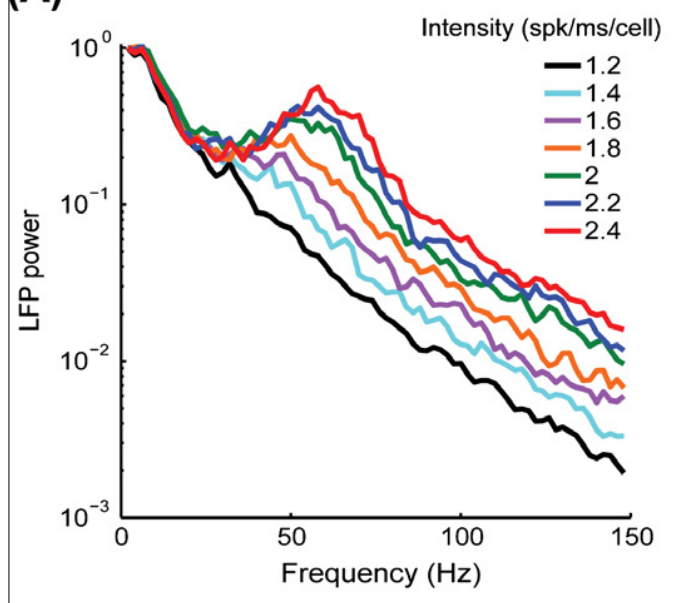

(B)

Periodic inputs

Input Amplitude: $1.6 \mathrm{spk} / \mathrm{ms} / \mathrm{cell}$

— Input Frequency: $4 \mathrm{~Hz}$

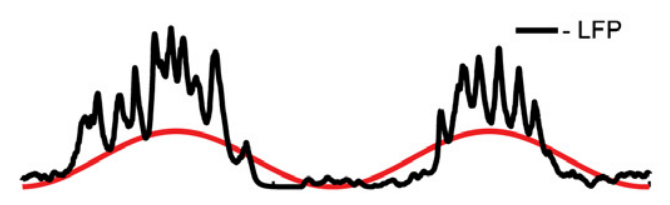

_ Input Frequency: $8 \mathrm{~Hz}$

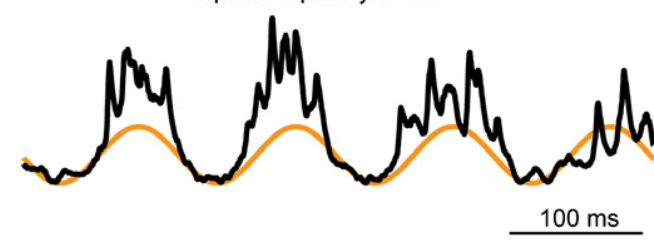

(C)

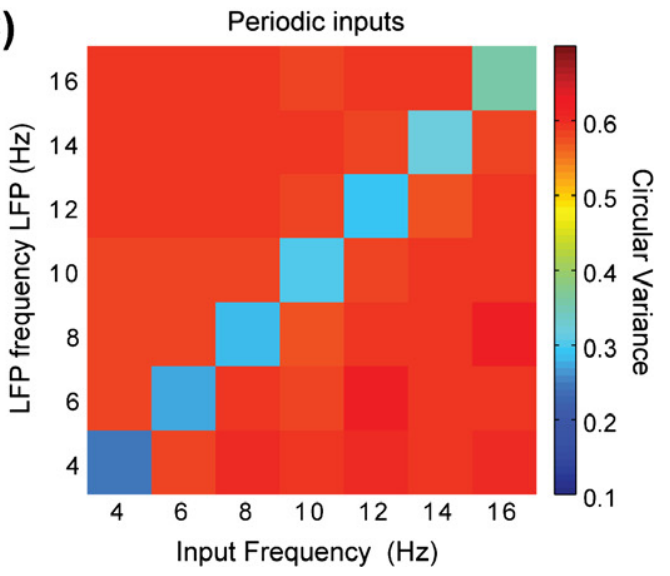

Fig. 5. Information processing in a recurrent network model injected with simple inputs (A) LFP spectral modulation when the network was injected with constant inputs of different intensity (see legend). The peak of the modulation occurred in the range [ $50-100 \mathrm{~Hz}$ ] in the gamma band. (B) LFP dynamics when the network was injected with low-frequency periodic inputs (see legend). The low frequency component of the network LFP entrained to the input. The network also displayed cross frequency coupling between high frequency power and low frequency phase. (C) The circular variance over time of the difference between the considered frequency component of the LFP and the considered frequency component of the input (each bandpassed in $2 \mathrm{~Hz}$ wide bands - see Section 2). This shows that entrainment became weaker at higher input frequencies. Panels ( $A$ and $C$ ) modified from Mazzoni et al. (2008), panel (B) modified from Panzeri et al. (2010) with permission. one (Fig. 7A): significant information was conveyed at low frequencies, and in the gamma band and above, with the two information peaks separated by an interval of non-informative frequencies. Also the combined information was similar to the one found experimentally: frequencies above $50 \mathrm{~Hz}$ were redundant with each other and independent from the informative frequencies at the low end of the spectrum (Fig. 7B and C). This was a consequence of the fact that input features encoded in the two bands, the intensity and the power at low frequencies, varied independently from scene to scene (Mazzoni et al., 2008).

In sum, the model reproduced well the experimental data and suggested answers to the two questions about the underlying mechanism asked at the beginning of the section: (i) the low frequencies convey information about the low frequency components in the input, and the gamma frequencies convey information about the strength of the input; (ii) encoding at low frequencies occurs through entrainment of the local neural activity to the external stimuli, encoding at gamma frequencies occurs through modulations of locally generated rhythms. Note that the presence of two streams of information could only be detected using naturalistic inputs which display large variations in intensity and a power spectrum peaking at low frequencies.

\subsection{A recurrent networks with slow dynamic inputs naturally reproduce cross-frequency phase-power coupling}

Another interesting finding of the dynamics of recurrent networks under naturalistic time-varying stimulation was that these networks presented cross-frequency phase-power coupling, i.e. the power of fast rhythms - such as the gamma rhythm - depended upon the phase of slower rhythms (Mazzoni et al., 2010). This phenomenon has also been consistently observed in neocortex (Canolty et al., 2006; He et al., 2010; Jensen and Colgin, 2007; Whittingstall and Logothetis, 2009) and hippocampus (Bragin et al., 1995; Lisman, 2005) and was proposed to be central for a number of cognitive and sensory processes (Jensen and Colgin, 2007; Lisman, 2005; Schroeder and Lakatos, 2009).

The presence of cross-frequency phase-power coupling in our simulated network is illustrated in Fig. 5B: when slow input fluctuations are present (such as the slow sinusoidal inputs of Fig. 5B), the gamma LFP power is higher when the input reaches a local maximum in time and thus (because of entrainment between low frequency LFPs and inputs) the LFP phase reaches a value signaling a maximum of the low frequency LFP (Mazzoni et al., 2010)). As a result of this simple mechanism, we found that, when stimulating the network with the naturalistic LGN input, the phase of delta range oscillations (which dominated the fluctuations in the naturalistic input, see Fig. 6A), significantly modulated the power of gamma oscillations (Fig. 8). This cross-frequency phase-power coupling was also found in experimental V1 recordings (Mazzoni et al., 2010), and the model could quantitatively account for the observed amount of coupling in real data (Fig. 8). These results suggest that the interplay between fast oscillation reflecting excitatory-inhibitory recurrent interactions and the slow oscillations reflecting changes in the external word contributes to the origin of the well documented phenomenon of cross-frequency coupling.

\section{Coding of visual information by the phase of firing}

The previous sections showed that during stimulation with naturalistic dynamics both visual cortex and recurrent network models develop slow fluctuations which are informative about the external world and can be measured by recording LFP. An interesting ques- 
(B)
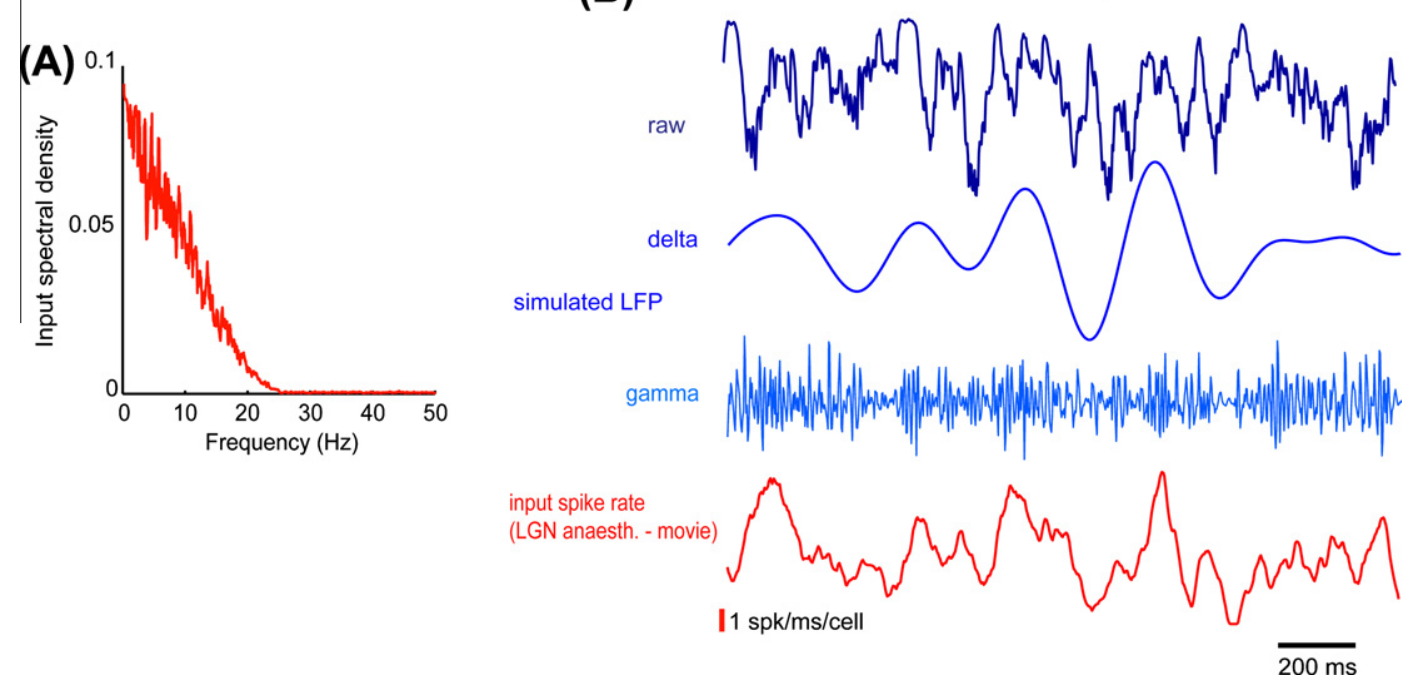

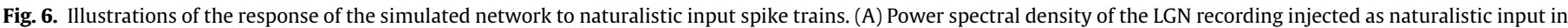

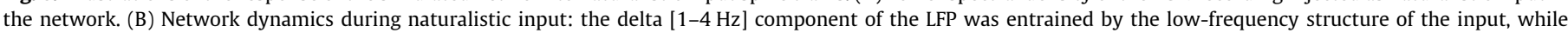

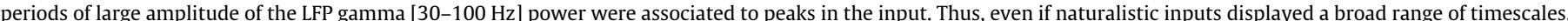

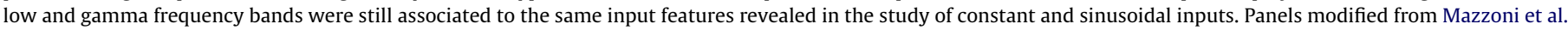
(2010) with permission.

tion is whether these fluctuations provide an internal temporal frame which can be used to reference spike times and increase the information that can be extracted from them. The existence of this type of spike timing coding, called phase of firing, has received considerable attention in recent years. Evidence has been reported that spatial-navigation and memory-related structures encode some information by phase of firing (Huxter et al., 2003; Lee et al., 2005; O'Keefe and Recce, 1993). However, the extent to which firing rate and phase encode genuinely different information is still debated (Harris, 2005; Harris et al., 2002; Mehta et al., 2002). Furthermore, the research has focused so far mainly on the role of phase of firing in the hippocampus, without clarifying whether phase coding represents a fundamental code for cortical information transmission used already in primary sensory areas.

In a recent study, we used the V1 recordings of LFPs and spikes in response to movie stimuli to investigate whether such phase of firing codes carry information about complex naturalistic visual stimuli (Montemurro et al., 2008). We found that the presentation of naturalistic color movies elicited reliable responses across trials both for the spikes and for the delta-band [1-4 Hz] LFP phase (Fig. 9A top rows). To visualize how LFP phases were modulated by the movie, we divided the phase range into four equi-spaced quadrants and labeled each with a different color (Fig. 9A). It was apparent that the [1-4 Hz] LFP phase also encoded the movie, because the phase values were modulated by the movie time, and this modulation was reliable across trials at several times during the movie (Montemurro et al., 2008). Visual inspection of the data suggested the LFP phase at which spike were fired allowed to disambiguate different movie scenes eliciting the same firing rate (Fig. 9A bottom rows), suggesting that the phase of firing carried visual information not available in the spike rates. This point was investigated in detail using information theory. We found (Fig. 9B) that the phase of the low frequency [1-4 Hz] LFP at which spikes were fired carried 55\% more information than spike counts about the movie segment being shown. Labeling the spikes with the phase of higher frequencies LFPs increased the information by a much smaller amount, suggesting that spike times are particularly informative with respect to slow (rather than fast) LFP fluctuations. In another study, the phase of firing with respect to [4$8 \mathrm{~Hz}$ ] LFPs was found to carry large amount of information about complex natural sounds in auditory cortex (Kayser et al., 2009), suggesting a general role of such code in representing sensory stimuli with complex, naturalistic dynamics. Slow fluctuations in the excitability of the local network can be measured by considering low frequency LFPs (Buzsaki and Draguhn, 2004; Logothetis, 2002; Schroeder and Lakatos, 2009), and the phase of such LFPs reflects the timing of changes in the state of the network and in its excitability. Thus, the increased information available in the phase of firing with respect to low frequency LFP fluctuations could be interpreted as suggesting that knowledge of the network state generating a spiking response would increase the information that the spiking response carries.

We analyzed the dynamics of the phase of firing code studying the responses of our local cortical network model when injected with naturalistic inputs (see Section 2.2). Each input was injected 100 times with different outcomes of the process representing the cortical spontaneous activity in order to study the reliability of the LFP and the amount of information conveyed by the phase of firing code (see Section 2.3). We bandpassed the network LFP response into different frequency bands (see Section 2.1.1) and we found that the reliability of the LFP phase was high for low frequencies and reached at $\sim 30 \mathrm{~Hz}$ a minimum level that was stable for higher frequencies. The same dynamics was observed in the experimental data (Montemurro et al., 2008), even if frequencies above $30 \mathrm{~Hz}$ were more reliable in simulations than in recordings. We built the phase of firing code considering the cumulative spiking activity of small groups of neurons (from 1 to 10), since this is the order of magnitude of the units available from single electrode recordings as those used in Montemurro et al. (2008). We found that the gain in information of the phase of firing relatively to the spike rate grew linearly with the average firing rate, i.e. the amount of information that the phase label added to each spike was relatively stable. As observed in Montemurro et al. (2008), the phase of firing gain was larger for low frequencies (Fig. 9C) even if it never reached zero in simulations because of the higher reliability of high frequency bands. Notably, very good quantitative agreement with experimental data was obtained when considering the cumulative activity of few excitatory units with a total average firing rate of 5-10 spikes/s (Fig. 9C), in the range of typical values of rates recorded from a single extracellular electrode. 

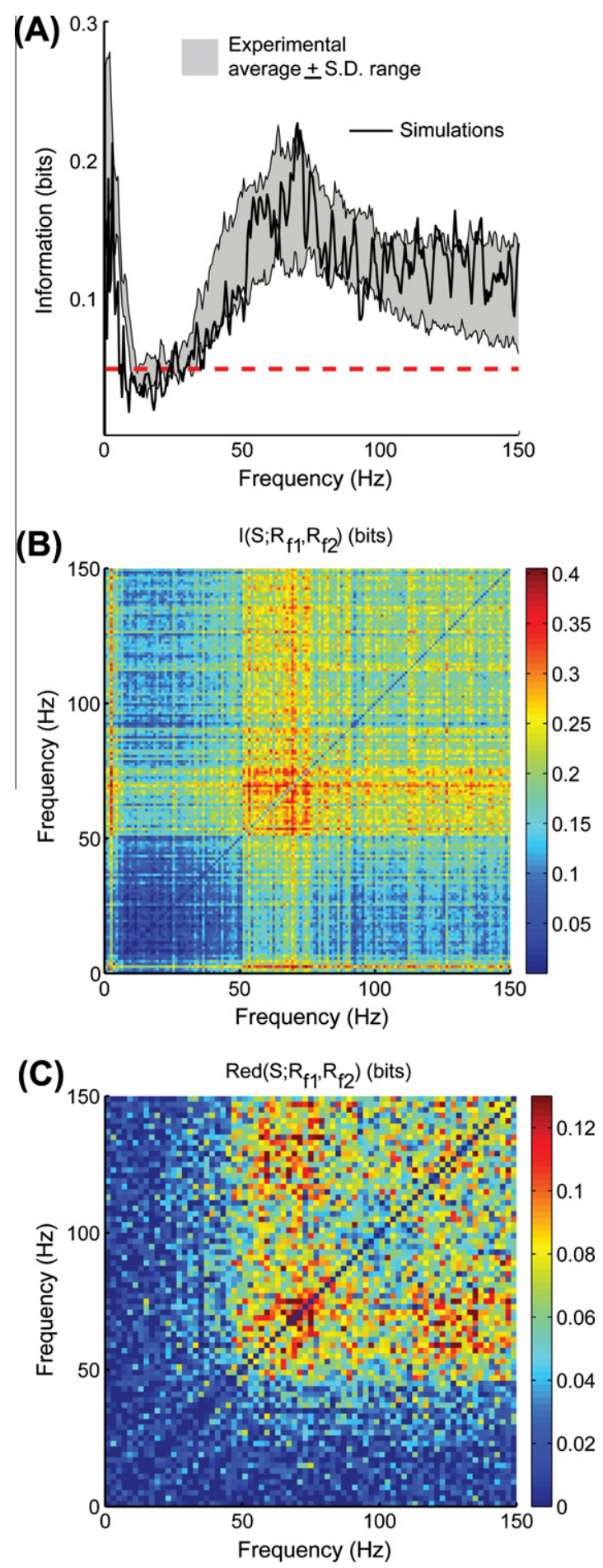

Fig. 7. The information carried by LFP power in a recurrent network model injected with naturalistic inputs. (A) Information about different parts of the dynamic input stimulus carried by the LFP power for both experimental recordings (gray area, representing the mean \pm STD area across the different channels of session A98) and simulations (black line). The red dashed line represents the $p=0.05$ (bootstrap test) significance line of information values. (B) Joint information and (C) redundancy of simulated LFP power for all frequency pairs. Results are consistent with experimental results in Fig. 4: (i) there were two peaks of information, one for low frequencies and one in the [50-100 Hz] frequency range in the gamma band; (ii) low frequencies and gamma frequencies carried independent information (iii) there was high redundancy within the gamma range. Panels modified from Mazzoni et al. (2008) with permission.

Overall, these results suggest that (i) low frequency LFP phase and spiking activity in the primary visual cortex convey independent information about naturalistic stimuli; (ii) a phase of firing

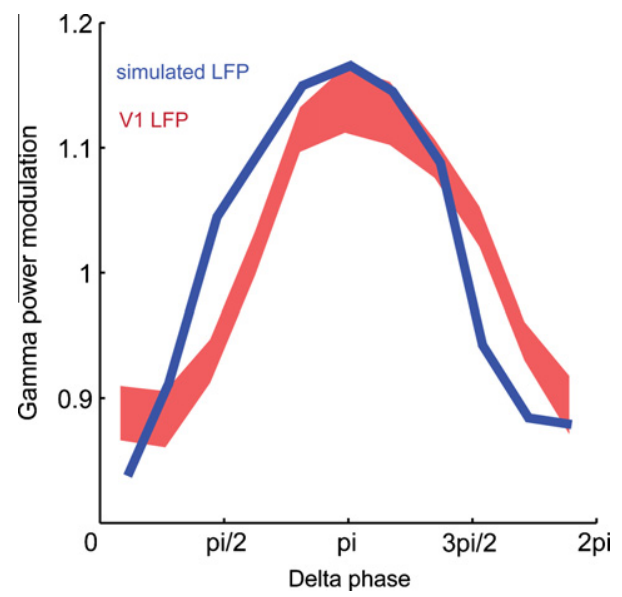

Fig. 8. Cross frequency phase-power coupling in experiment and simulations. The plot shows the modulation of gamma amplitude as a function of delta phase in the LFP recorded from primary visual cortex (red shaded area representing mean and SEM across recording sessions) compared to the modulation of the simulated LFP obtained in response to a naturalistic input (blue line, mean across all simulation points). Panel modified from Mazzoni et al. (2010) with permission.

code carries indeed more information than the spike rate alone (and is also more robust to noise); (iii) this dynamics can be studied in the same framework used to analyze the phenomena described in the previous sections, and in particular by the means of the same recurrent LIF network.

\section{Discussion}

In this article we presented the progress that we made in characterizing empirically the frequency ranges used by primary cortical neural populations to represent naturalistic sensory information and in using models to test quantitatively specific hypotheses about the biological mechanisms generating these neural representations.

\subsection{Multiplexing and sensory coding}

Our main empirical result is that, out of the very wide range of frequencies expressed by the activity of the primary visual cortex, only two frequency ranges carry significant information. The first consists in low frequency $(<10 \mathrm{~Hz}$ ) fluctuations, and the second consists in gamma range oscillations. Low frequency fluctuations are decoupled and independent from gamma oscillations and spiking activity, whereas gamma oscillations carry information similar to that of spiking activity. Our models suggest that this information independence stems from the difference in network mechanisms originating the activity in the different frequencies and in the different nature of the information encoded. In particular, our models suggest that recurrent cortical excitatory-inhibitory networks encode the strength of the input to the network as gamma-range oscillations generated by inhibitory-excitatory neural interactions, and encode slow dynamic features of the input into slow LFP fluctuations that are entrained to the slow fluctuations of the naturalistic stimuli.

Taken together, these findings suggest that a key strategy used by cortical networks to cope with the challenges of representing the complexity of the natural environment is to use a multiplexing strategy to encode simultaneously different types of information at different time scales and so enhance the information capacity of cortical columns (Panzeri et al., 2010). Evidence is now accumulating that a multiplexing strategy, suggested in earlier seminal work (Bullock, 1997; Lisman, 2005) is key for the brain to represent the complexity of changing environments (Fairhall et al., 2001; Lund- 
(A)
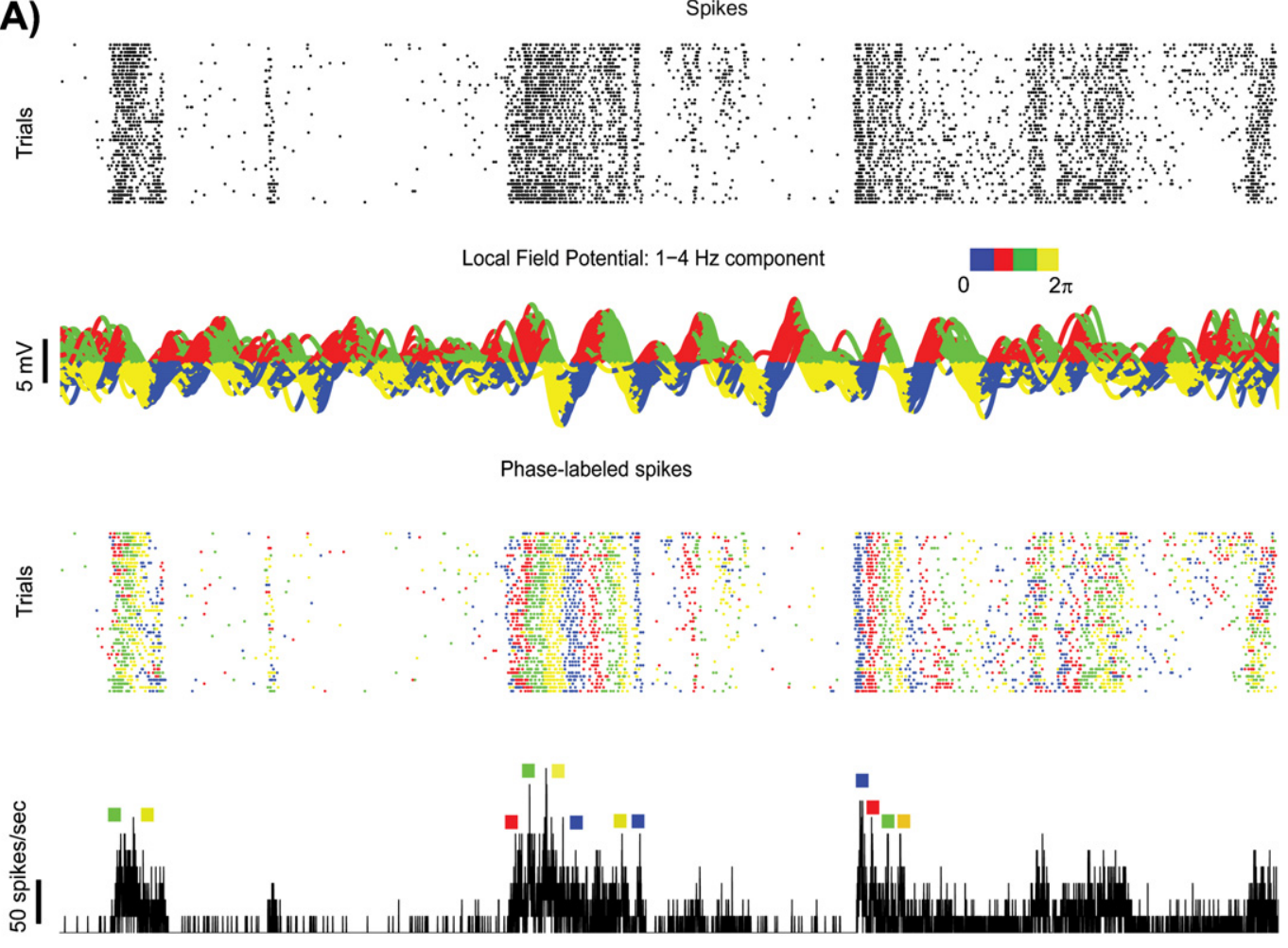

$1 \mathrm{sec}$
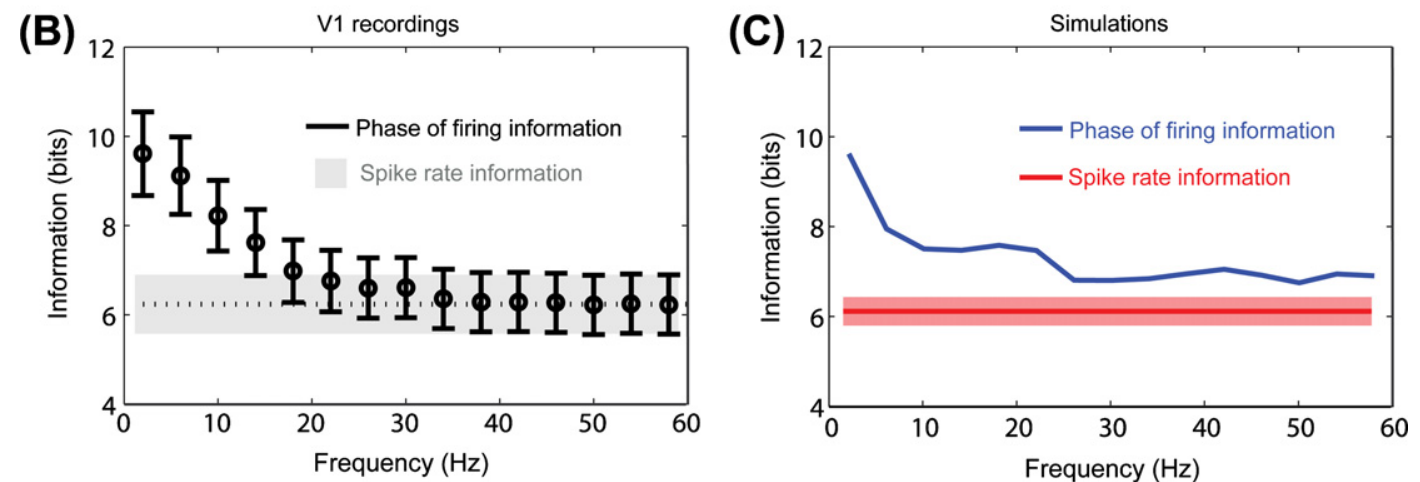

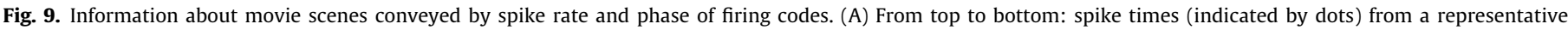

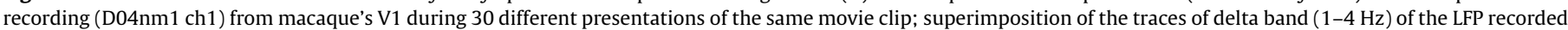

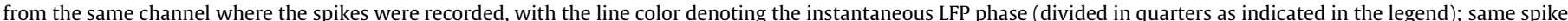

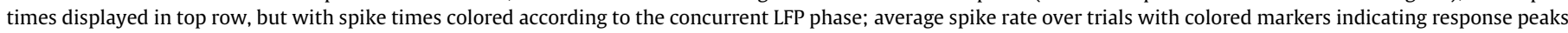

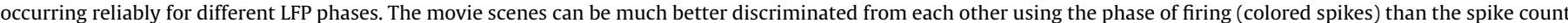

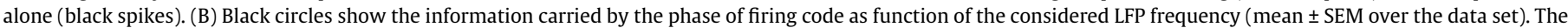

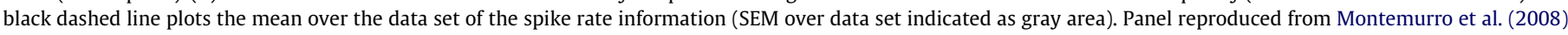

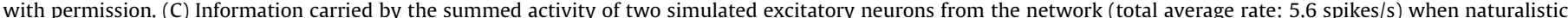

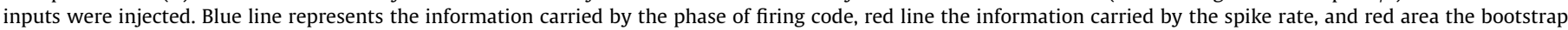
significance $(p<0.05)$ obtained with 40 permutations of the phase.

strom and Fairhall, 2006) and of the information relevant for behavior (Schyns et al., 2011). These findings have also potential implications for the development of Brain Machine Interfaces (Donoghue, 2008; Nicolelis and Lebedev, 2009), because decoding based on multiple time scales may be used to enhance the amount of information that can be extracted by each single electrode.

\subsection{Implications of the model prediction that information in low} frequency LFPs arises in part by entrainment to slow stimulus features

Our modeling work suggested that the information about naturalistic movies carried by low frequency LFPs results from entrain- ment to the low frequency components of the time evolution of naturalistic stimuli. This model prediction is consistent with results from the auditory system, which show that low frequency LFPs entrain to sound features during the presentation of complex naturalistic sounds (Chandrasekaran et al., 2010).

An experimentally testable prediction of this model, which we are currently investigating, is that low frequency LFPs in primary visual cortex should entrain to the frame by frame changes of one or more image features (such as contrast or orientation) displayed in the receptive field of the considered recording sites during movie presentation. This is because the latter should somehow reflect the time course of the input to the local cortical network, as these fea- 
tures influence the firing of geniculate neurons and of other cortical areas.

Natural visual stimuli are characterized by both "what" aspects (image properties such as contrast or orientation which are defined by the relationship between visual signals simultaneously presented at different points in space) and "when" aspects, describing the temporal variations of the various image features. Although temporal information is crucial for sensory processing, our understanding of stimulus processing is currently biased toward the representation of non-temporal aspects (Buonomano and Maass, 2009). A potentially interesting implication of the hypothesis that low frequency LFPs entrain to the slow stimulus dynamics is that they may provide a way to encode information about the temporal structure of natural visual stimuli in the low temporal frequency range $(<10 \mathrm{~Hz})$ in which these stimuli have the most power and information (Dan et al., 1996; Geisler, 2008).

\subsection{Partial decoupling of the information carried by gamma oscillations and spike rates during naturalistic stimulation}

We investigated whether LFPs and spikes convey redundant or independent stimulus information at particular frequencies, and we found that in primary visual cortex of anaesthetized macaques the power of gamma range LFPs was largely (but not completely) redundant to the spike rate, indicating that spike rates and gamma power are similar but can also decouple in some condition during naturalistic stimulation. We note that in our simple model of a local randomly connected recurrent network with balanced excitation and inhibition, spike rate and power should be largely coupled, because in these model networks higher input excitation corresponds to higher gamma power and higher output spike rate (Brunel and Wang, 2003). For example, an increase in contrast within the receptive field is likely to elicit an increase of both spike rate and gamma power. It is therefore interesting to consider the reasons and mechanisms by which this partial decoupling may arise. Thiele and Gieselmann (2008) reported that in V1 of awake macaques an increase in the size of the visual stimulus was associated to a decrease in the spiking activity and to an increase in the LFP power, particularly in the gamma band. The decoupling occurred when the stimulus borders reached the surround of the receptive field: the inhibition from the receptive field surround mechanisms suppressed the firing activity while contributed to strengthen the LFP gamma oscillations. Thus, one possibility is that the partial decoupling between gamma power and spike rate that we observed is caused by objects present in natural movies changing their size across time, and this could be modeled by a differential recruitment of excitatory and inhibitory populations during the movie. A second factor contributing to decoupling in our data may be the one proposed in an interesting modeling study by Deco and colleagues (2008), which revealed the mechanisms of decoupling between gamma power and spike rates in models of networks made of pools of interacting neurons. Their results suggest that the interplay of different networks, which was not considered in our own simulations, may also contribute to the partial decoupling between gamma power and spike rate which we observed.

\subsection{Statistical validation of models and estimation of model parameters}

In principle, models like the ones presented here are not only useful to provide a potential explanation of the network phenomena generating neural representations, but also to estimate the changes across different stimulation conditions of important parameters of the network (such as the balance of excitation and inhibition) which would be difficult or impossible to measure directly from extracellular recordings. So far, we estimated network parameters by first fixing them from plausible literature values, and then fine tune them by hand to obtain a good fit with the experimental data, as measured by chi square statistics. However, in principle, methods such as Dynamic Expectation Maximization (Friston et al., 2008) or Bayesian estimation (Friston, 2002) could be used to obtain a much more rigorous correspondence between model and data and estimate the best fit parameters as well as the confidence of parameter estimation. However, a practical problem is that these statistical procedures require integrating the network dynamics and produce a numerical output over a wide range of possible different parameter values, and this is not feasible with simulations like the one described above, because of the high dimensional parameter space and because of the long time currently taken to run the analysis (several hours per parameter setting on a state of the art PC). A solution to this problem is to obtain analytical approximations of the model network variables that can be measured experimentally (such as the LFP or spike rate spectrum or the spectral LFP information) which are valid in a given network regime (such as the weakly synchronized one) and then use these approximations to estimate the network parameters by means of the statistical techniques described above. The analytical methods consist in computing the dynamics of the instantaneous firing rate of both excitatory and inhibitory neurons in the presence of time-dependent inputs, using linear response theory (Ledoux and Brunel, 2011). This allows in turn the computation of the LFP spectrum and spectral LFP information, and the use of these analytical expressions to fit the experimental data. We are currently working toward this goal.

\section{Acknowledgements}

This work was supported by the BMI project of the Italian Institute of Technology, the Max Planck Society, the Compagnia di San Paolo, and was part of the research program of the Bernstein Center for Computational Neuroscience, Tübingen, funded by the German Federal Ministry of Education and Research (BMBF; FKZ: 01GQ1002).

\section{References}

Amit, J.A., Brunel, N., Tsodyks, M.V., 1994. Correlations of cortical Hebbian reverberations: theory versus experiment. J. Neurosci. 14 (11), 6435-6445.

Baddeley, R., Abbott, L.F., Booth, M.C., Sengpiel, F., Freeman, T., Wakeman, E.A., Rolls, E.T., 1997. Responses of neurons in primary and inferior temporal visual cortices to natural scenes. Proc. Biol. Sci. 264 (1389), 1775-1783.

Bedard, C., Kroger, H., Destexhe, A., 2006. Does the $1 / f$ frequency scaling of brain signals reflect self-organized critical states? Phys. Rev. Lett. 97 (11), 118102.

Belitski, A., Gretton, A., Magri, C., Murayama, Y., Montemurro, M.A., Logothetis, N.K. Panzeri, S., 2008. Low-frequency local field potentials and spikes in primary visual cortex convey independent visual information. J. Neurosci. 28 (22), 56965709

Belitski, A., Panzeri, S., Magri, C., Logothetis, N.K., Kayser, C., 2010. Sensory information in local field potentials and spikes from visual and auditory cortices: time scales and frequency bands. J. Comput. Neurosci. 29 (3), 533-545.

Benucci, A., Ringach, D.L., Carandini, M., 2009. Coding of stimulus sequences by population responses in visual cortex. Nat. Neurosci. 12 (10), 1317-1324.

Berens, P., Keliris, G.A., Ecker, A.S., Logothetis, N.K., Tolias, A.S., 2008. Comparing the feature selectivity of the gamma-band of the local field potential and the underlying spiking activity in primate visual cortex. Front. Syst. Neurosci. 2, 2.

Bragin, A., Jando, G., Nadasdy, Z., Hetke, J., Wise, K., Buzsaki, G., 1995. Gamma (40$100 \mathrm{~Hz}$ ) oscillation in the hippocampus of the behaving rat. J. Neurosci. 15 (1 Pt 1), 47-60.

Breakspear, M., Jirsa, V., Deco, G., 2010. Computational models of the brain: from structure to function. Neuroimage 52 (3), 727-730.

Brunel, N., 2000. Dynamics of sparsely connected networks of excitatory and inhibitory spiking neurons. J. Comput. Neurosci. 8 (3), 183-208.

Brunel, N., Wang, X.J., 2003. What determines the frequency of fast network oscillations with irregular neural discharges? I. Synaptic dynamics and excitation-inhibition balance. J. Neurophysiol. 90 (1), 415-430.

Buehlmann, A., Deco, G., 2008. The neuronal basis of attention: rate versus synchronization modulation. J. Neurosci. 28 (30), 7679-7686.

Bullock, T.H., 1997. Signals and signs in the nervous system: the dynamic anatomy of electrical activity is probably information-rich. Proc. Natl. Acad. Sci. USA 94 (1), 1-6. 
Buonomano, D.V., Maass, W., 2009. State-dependent computations: spatiotemporal processing in cortical networks. Nat. Rev. Neurosci. 10 (2), 113-125.

Buszaki, G., 2006. Rhythms of the Brain. Oxford University Press, New York.

Buzsaki, G., Draguhn, A., 2004. Neuronal oscillations in cortical networks. Science 304 (5679), 1926-1929.

Canolty, R.T., Edwards, E., Dalal, S.S., Soltani, M., Nagarajan, S.S., Kirsch, H.E., Berger, M.S., Barbaro, M.N., Knight, R.T., 2006. High gamma power is phase-locked to theta oscillations in human neocortex. Science 313 (5793), 1626-1628.

Chandrasekaran, C., Turesson, H.K., Brown, C.H., Ghazanfar, A.A., 2010. The influence of natural scene dynamics on auditory cortical activity. J. Neurosci. 30 (42), 13919-13931.

Dan, Y., Atick, J.J., Reid, R.C., 1996. Efficient coding of natural scenes in the lateral geniculate nucleus: experimental test of a computational theory. J. Neurosci. 16 (10), 3351-3362.

David, F.O., Hugues, E., Cenier, T., Fourcaud-Trocme, N., Buonviso, N., 2009. Specific entrainment of mitral cells during gamma oscillation in the rat olfactory bulb. PLoS Comput. Biol. 5 (10), e1000551.

Deco, G., Jirsa, V.K., Robinson, P.A., Breakspear, M., Friston, K., 2008. The dynamic brain: from spiking neurons to neural masses and cortical fields. PLoS Comput. Biol. 4 (8), e1000092.

Derrington, A.M., Lennie, P., 1984. Spatial and temporal contrast sensitivities of neurones in lateral geniculate nucleus of macaque. J. Physiol. 357, $219-240$.

Desbordes, G., Jin, J., Weng, C., Lesica, N.A., Stanley, G.B., Alonso, J.M., 2008. Timing precision in population coding of natural scenes in the early visual system. PLoS Biol. 6 (12), e324.

Donoghue, J.P., 2008. Bridging the brain to the world: a perspective on neural interface systems. Neuron 60 (3), 511-521.

Douglas, R.J., Martin, K.A., 1991. A functional microcircuit for cat visual cortex. J. Physiol. (Lond.) 440, 735-769.

Fairhall, A.L., Lewen, G.D., Bialek, W., de Ruyter Van Steveninck, R.R., 2001. Efficiency and ambiguity in an adaptive neural code. Nature 412 (6849), 787792.

Felsen, G., Dan, Y., 2005. A natural approach to studying vision. Nat. Neurosci. 8 (12), 1643-1646.

Fisher, N.I., 1993. Statistical Analysis of Circular Data. Cambridge University press, Cambridge.

Fix, J., Rougier, N., Alexandre, F., 2007. From physiological principles to computational models of the cortex. J. Physiol. (Paris) 101 (1-3), 32-39.

Friedman-Hill, S., Maldonado, P.E., Gray, C.M., 2000. Dynamics of striate cortical activity in the alert macaque: I. Incidence and stimulus-dependence of gammaband neuronal oscillations. Cereb. Cortex 10 (11), 1105-1116.

Friston, K.J., 2002. Bayesian estimation of dynamical systems: an application to fMRI. Neuroimage 16 (2), 513-530.

Friston, K.J., Trujillo-Barreto, N., Daunizeau, J., 2008. DEM: a variational treatment of dynamic systems. Neuroimage 41 (3), 849-885.

Geisler, W.S., 2008. Visual perception and the statistical properties of natural scenes. Annu. Rev. Psychol. 59, 167-192.

Gieselmann, M.A., Thiele, A., 2008. Comparison of spatial integration and surround suppression characteristics in spiking activity and the local field potential in macaque V1. Eur. J. Neurosci. 28 (3), 447-459.

Gil, Z., Amitai, Y., 1996. Properties of convergent thalamocortical and intracortical synaptic potentials in single neurons of neocortex. J. Neurosci. 16 (20), 65676578.

Goense, J.B., Logothetis, N.K., 2008. Neurophysiology of the BOLD fMRI signal in awake monkeys. Curr. Biol. 18 (9), 631-640.

Goense, J.B., Ku, S.P., Merkle, H., Tolias, A.S., Logothetis, N.K., 2008. FMRI of the temporal lobe of the awake monkey at $7 \mathrm{~T}$. Neuroimage 39 (3), 1081-1093.

Gupta, A., Wang, Y., Markram, H., 2000. Organizing principles for a diversity of GABAergic interneurons and synapses in the neocortex. Science 287 (5451), 273-278.

Gustafsonn, B., 1984. Afterpotentials and transduction properties in different types of central neurones. Arch. Ital. Biol. 122, 17-30.

Harada, Y., Takahashi, T., 1983. The calcium component of the action potential in spinal motoneurons of the rat. J. Physiol. (Lond.) 335, 89-100.

Harris, K.D., 2005. Neural signatures of cell assembly organization. Nat. Rev. Neurosci. 6 (5), 399-407.

Harris, K.D., Henze, D.A., Hirase, H., Leinekugel, X., Dragoi, G., Czurko, A., Buzsaki, G., 2002. Spike train dynamics predicts theta-related phase precession in hippocampal pyramidal cells. Nature 417 (6890), 738-741.

Hasson, U., Malach, R., Heeger, D.J., 2009. Reliability of cortical activity during natural stimulation. Trends Cogn. Sci. 14 (1), 40-48.

He, B.J., Zempel, J.M., Snyder, A.Z., Raichle, M.E., 2010. The temporal structures and functional significance of scale-free brain activity. Neuron 66 (3), 353-369.

Henrie, J.A., Shapley, R., 2005. LFP power spectra in V1 cortex: the graded effect of stimulus contrast. J. Neurophysiol. 94 (1), 479-490.

Huxter, J., Burgess, N., O'Keefe, J., 2003. Independent rate and temporal coding in hippocampal pyramidal cells. Nature 425 (6960), 828-832.

Jensen, O., Colgin, L.L., 2007. Cross-frequency coupling between neuronal oscillations. Trends Cogn. Sci. 11 (7), 267-269.

Jones, A.K., Qi, L.Y., Fujirawa, T., Luthra, S.K., Ashburner, J., Bloomfield, P., Cunningham, V.J., Itoh, M., Fukuda, H., Jones, T., 1991. In vivo distribution of opioid receptors in man in relation to the cortical projections of the medial and lateral pain systems measured with positron emission tomography. Neurosci. Lett. 126 (1), 25-28.
Kamondi, A., Acsady, L., Wang, X.J., Buzsaki, G., 1998. Theta oscillations in somata and dendrites of hippocampal pyramidal cells in vivo: activity-dependent phase-precession of action potentials. Hippocampus 8 (3), 244-261.

Katzner, S., Nauhaus, I., Benucci, A., Bonin, V., Ringach, D.L., Carandini, M., 2009 Local origin of field potentials in visual cortex. Neuron 61, 35-41.

Kayser, C., Konig, P., 2004. Stimulus locking and feature selectivity prevail in complementary frequency ranges of V1 local field potentials. Eur. J. Neurosci. 19 (2), 485-489.

Kayser, C., Montemurro, M.A., Logothetis, N.K., Panzeri, S., 2009. Spike-phase coding boosts and stabilizes information carried by spatial and temporal spike patterns. Neuron 61 (4), 597-608.

Lakatos, P., Shah, A.S., Knuth, K.H., Ulbert, I., Karmos, G., Schroeder, C.E., 2005. An oscillatory hierarchy controlling neuronal excitability and stimulus processing in the auditory cortex. J. Neurophysiol. 94 (3), 1904-1911.

Lakatos, P., Karmos, G., Mehta, A.D., Ulbert, I., Schroeder, C.E., 2008. Entrainment of neuronal oscillations as a mechanism of attentional selection. Science 320 (5872), 110-113.

Ledoux, E., Brunel, N., 2011. Dynamics of networks of excitatory and inhibitory neurons in response to time-dependent inputs. Front. Comput. Neurosci. 5.

Lee, H., Simpson, G.V., Logothetis, N.K., Rainer, G., 2005. Phase locking of single neuron activity to theta oscillations during working memory in monkey extrastriate visual cortex. Neuron 45 (1), 147-156.

Leppa, M., Korvenoja, A., Carlson, S., Timonen, P., Martinkauppi, S., Ahonen, J., Rosenberg, P.H., Aronen, H.J., Kalso, E., 2006. Acute opioid effects on human brain as revealed by functional magnetic resonance imaging. Neuroimage 31 (2), 661-669.

Lisman, J., 2005. The theta/gamma discrete phase code occuring during the hippocampal phase precession may be a more general brain coding scheme. Hippocampus 15 (7), 913-922.

Logothetis, N.K., 2002. The neural basis of the blood-oxygen-level-dependent functional magnetic resonance imaging signal. Philos. Trans. Roy. Soc. B - Biol. Sci. 357 (1424), 1003-1037.

Logothetis, N.K., 2003. The underpinnings of the BOLD functional magnetic resonance imaging signal. J. Neurosci. 23 (10), 3963-3971.

Logothetis, N.K., 2008. What we can do and what we cannot do with fMRI. Nature 453 (7197), 869-878.

Logothetis, N.K., Guggenberger, H., Peled, S., Pauls, J., 1999. Functional imaging of the monkey brain. Nat. Neurosci. 2 (6), 555-562.

Logothetis, N., Merkle, H., Augath, M., Trinath, T., Ugurbil, K., 2002. Ultra highresolution fMRI in monkeys with implanted RF coils. Neuron 35 (2), 227-242.

Logothetis, N.K., Murayama, Y., Augath, M., Steffen, T., Werner, J., Oeltermann, A., 2009. How not to study spontaneous activity. Neuroimage 45 (4), 1080-1089.

Lund, J.S., Yoshioka, T., Levitt, J.B., 1994. Substrates for Interlaminar Connection in Area V1 of Macaque Monkey Cerebral Cortex in Primary Visual Cortex in Primates. Plenum Press, New York.

Lundstrom, B.N., Fairhall, A.L., 2006. Decoding stimulus variance from a distributional neural code of interspike intervals. J. Neurosci. 26 (35), 90309037.

Luo, H., Poeppel, D., 2007. Phase patterns of neuronal responses reliably discriminate speech in human auditory cortex. Neuron 54 (6), 1001-1010.

Magri, C., Whittingstall, K., Singh, V., Logothetis, N.K., Panzeri, S., 2009. A toolbox for the fast information analysis of multiple-site LFP, EEG and spike train recordings. BMC Neurosci. 10, 81.

Markram, H., Lubke, J., Frotscher, M., Roth, A., Sakmann, B., 1997. Physiology and anatomy of synaptic connections between thick tufted pyramidal neurones in the developing rat neocortex. J. Physiol. 500 (Pt 2), 409-440.

Mazzoni, A., Broccard, F.D., Garcia-Perez, E., Bonifazi, P., Ruaro, M.E., Torre, V., 2007. On the dynamics of the spontaneous activity in neuronal networks. PLoS ONE 2 (5), e439.

Mazzoni, A., Panzeri, S., Logothetis, N.K., Brunel, N., 2008. Encoding of naturalistic stimuli by local field potential spectra in networks of excitatory and inhibitory neurons. PLoS Comput. Biol. 4 (12), e1000239.

Mazzoni, A., Whittingstall, K., Brunel, N., Logothetis, N.K., Panzeri, S., 2010. Understanding the relationships between spike rate and delta/gamma frequency bands of LFPs and EEGs using a local cortical network model. Neuroimage 52 (3), 956-972.

Mehta, M.R., Lee, A.K., Wilson, M.A., 2002. Role of experience and oscillations in transforming a rate code into a temporal code. Nature 417 (6890), 741-746.

Mitzdorf, U., 1985. Current source-density method and application in cat cerebral cortex: investigation of evoked potentials and EEG phenomena. Physiol. Rev. 65 (1), 37-100.

Montemurro, M.A., Panzeri, S., Maravall, M., Alenda, A., Bale, M.R., Brambilla, M., Petersen, R.S., 2007a. Role of precise spike timing in coding of dynamic vibrissa stimuli in somatosensory thalamus. J. Neurophysiol. 98 (4), 1871-1882.

Montemurro, M.A., Senatore, R., Panzeri, S., 2007b. Tight data-robust bounds to mutual information combining shuffling and model selection techniques. Neural Comput. 19 (11), 2913-2957.

Montemurro, M.A., Rasch, M.J., Murayama, Y., Logothetis, N.K., Panzeri, S., 2008 Phase-of-firing coding of natural visual stimuli in primary visual cortex. Curr. Biol. 18 (5), 375-380.

Murakami, S., Okada, Y., 2006. Contributions of principal neocortical neurons to magnetoencephalography and electroencephalography signals. J. Physiol. 575 (Pt 3), 925-936.

Muthukumaraswamy, S.D., Singh, K.D., Swettenham, J.B., Jones, D.K., 2010. Visual gamma oscillations and evoked responses: variability, repeatability and structural MRI correlates. Neuroimage 49 (4), 3349-3357. 
Nicolelis, A.L.M., Lebedev, M.A., 2009. Principles of neural ensemble physiology underlying the operation of brian-machine interfaces. Nat. Rev. Neurosci. 10, 530-540.

O’Keefe, J., Recce, M.L., 1993. Phase relationship between hippocampal place units and the EEG theta rhythm. Hippocampus 3 (3), 317-330.

Panzeri, S., Petroni, F., Petersen, R.S., Diamond, M.E., 2003. Decoding neuronal population activity in rat somatosensory cortex: role of columnar organization. Cereb. Cortex 13 (1), 45-52.

Panzeri, S., Senatore, R., Montemurro, M.A., Petersen, R.S., 2007. Correcting for the sampling bias problem in spike train information measures. J. Neurophysiol. 98 (3), 1064-1072.

Panzeri, S., Magri, C., Logothetis, N.K., 2008. On the use of information theory for the analysis of the relationship between neural and imaging signals. Magn. Reson. Imaging 26 (7), 1015-1025.

Panzeri, S., Brunel, N., Logothetis, N.K., Kayser, C., 2010. Sensory neural codes using multiplexed temporal scales. Trends Neurosci. 33 (3), 111-120.

Percival, D.B., Walden, A.T., 1993. Spectral Analysis for Physical Applications: Multitaper and Conventional Univariate Techniques. Cambridge University Press, Cambridge, UK.

Pettersen, K.H., Hagen, E., Einevoll, G.T., 2008. Estimation of population firing rates and current source densities from laminar electrode recordings. J. Comput. Neurosci. 24 (3), 291-313.

Pfeiffer, A., Pasi, A., Mehraein, P., Herz, A., 1982. Opiate receptor binding sites in human brain. Brain Res. 248 (1), 87-96.

Rasch, M.J., Gretton, A., Murayama, Y., Maass, W., Logothetis, N.K., 2008. Inferring spike trains from local field potentials. J. Neurophysiol. 99, 1461-1476.

Ray, S., Maunsell, J.H., 2010. Differences in gamma frequencies across visual cortex restrict their possible use in computation. Neuron 67 (5), 885-896.

Reinagel, P., 2001. How do visual neurons respond in the real world? Curr. Opin. Neurobiol. 11 (4), 437-442.

Rust, N.C., Movshon, J.A., 2005. In praise of artifice. Nat. Neurosci. 8 (12), $1647-$ 1650.

Schroeder, C.E., Lakatos, P., 2009. Low-frequency neuronal oscillations as instruments of sensory selection. Trends Neurosci. 32 (1), 9-18.

Schyns, P.G., Thut, G., Gross, J., 2011. Cracking the code of oscillatory activity. PLoS Biol. 9 (5), e1001064
Senkowski, D., Gomez-Ramirez, M., Lakatos, P., Wylie, G.R., Molholm, S., Schroeder, C.E., Foxe, J.J., 2007. Multisensory processing and oscillatory activity: analyzing non-linear electrophysiological measures in humans and simians. Exp. Brain Res. 177 (2), 184-195.

Sereno, M.E., Trinath, T., Augath, M., Logothetis, N.K., 2002. Three-dimensional shape representation in monkey cortex. Neuron 33 (4), 635-652.

Shapley, R., Kaplan, E., Soodak, R., 1981. Spatial summation and contrast sensitivity of $X$ and $Y$ cells in the lateral geniculate nucleus of the macaque. Nature 292, 543-545.

Simoncelli, E.P., 2003. Vision and the statistics of the visual environment. Curr. Opin. Neurobiol. 13 (2), 144-149.

Smyth, D., Willmore, B., Baker, G.E., Thompson, I.D., Tolhurst, D.J., 2003. The receptive-field organization of simple cells in primary visual cortex of ferrets under natural scene stimulation. J. Neurosci. 23 (11), 4746-4759.

Swettenham, J.B., Muthukumaraswamy, S.D., Singh, K.D., 2009. Spectral properties of induced and evoked gamma oscillations in human early visual cortex to moving and stationary stimuli. J. Neurophysiol. 102 (2), 1241-1253.

Talbot, J.D., Marrett, S., Evans, A.C., Meyer, E., Bushnell, M.C., Duncan, G.H., 1991. Multiple representations of pain in human cerebral cortex. Science 251 (4999), 1355-1358.

Treves, A., Panzeri, S., Rolls, E.T., Booth, M., Wakeman, E.A., 1999. Firing rate distributions and efficiency of information transmission of inferior temporal cortex neurons to natural visual stimuli. Neural Comput. 11 (3), 601-632.

Tuckwell, H.C., 1988. Introduction to Theoretical Neurobiology. Cambridge University Press, Cambridge, UK.

de Ruyter van Steveninck, R.R., Lewen, G.D., Strong, S.P., Koberle, R., Bialek, W., 1997. Reproducibility and variability in neural spike trains. Science 275 (5307), 18051808 .

Whittingstall, K., Logothetis, N.K., 2009. Frequency-band coupling in surface EEG reflects spiking activity in monkey visual cortex. Neuron 64 (2), 281-289.

Zappe, A.C., Uludag, K., Logothetis, N.K., 2008a. Direct measurement of oxygen extraction with fMRI using 6\% CO2 inhalation. Magn. Reson. Imaging 26 (7), 961-967.

Zappe, A.C., Uludag, K., Oeltermann, A., Ugurbil, K., Logothetis, N.K., 2008b. The influence of moderate hypercapnia on neural activity in the anesthetized nonhuman primate. Cereb. Cortex 18 (11), 2666-2673. 